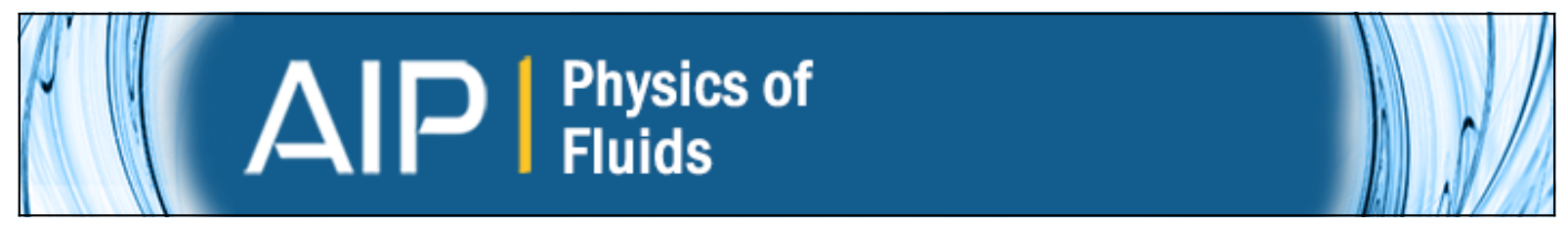

Sound-ultrasound interaction in bubbly fluids: Theory and possible applications

D. B. Khismatullin and I. Sh. Akhatov

Citation: Physics of Fluids (1994-present) 13, 3582 (2001); doi: 10.1063/1.1416502

View online: http://dx.doi.org/10.1063/1.1416502

View Table of Contents: http://scitation.aip.org/content/aip/journal/pof2/13/12?ver=pdfcov

Published by the AIP Publishing

Copyright by the American Institute of Physics. Sound-

ultrasound interaction in bubbly fluids: Theory and possible

applications. Khismatullin, D. B. and Akhatov, I. Sh., Physics

of Fluids (1994-present), 13, 3582-3598 (2001), DOI:http://

dx.doi.org/10.1063/1.1416502

AlP Re-register for Table of Content Alerts

Create a profile.

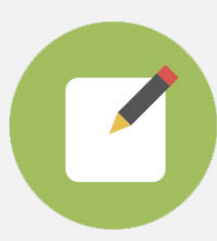




\title{
Sound-ultrasound interaction in bubbly fluids: Theory and possible applications
}

\author{
D. B. Khismatullin a) \\ Department of Aerospace and Mechanical Engineering, Boston University, 110 Cummington Street, \\ Boston, Massachusetts 02215 \\ I. Sh. Akhatov \\ Institute of Mechanics, Ufa (Bashkortostan) Branch of Russian Academy of Sciences, 12 K. Marx Street, \\ Ufa 450000, Russia
}

(Received 13 November 2000; accepted 7 September 2001)

\begin{abstract}
The interaction between sound and ultrasound waves in a weakly compressible viscous liquid with gas bubbles is considered. Using the method of multiple scales one- and two-dimensional nonlinear interaction equations are derived. The degeneracy of the interaction is found in bubbly fluids. This phenomenon lies in the fact that the interaction coefficients vanish at a certain frequency of ultrasound. We demonstrate that the integrable Davey-Stewartson I (DSI) system of equation can describe the two-dimensional sound-ultrasound evolution. The DSI equations are remarkable by their solutions referred to as dromions. In bubbly fluids the dromion represents the localized focused ultrasound wave which can alter the direction of its motion under changes in the boundary conditions for the sound wave. The condition of singular focusing of ultrasound in bubbly fluids is obtained. By numerical analysis of the interaction models, we reveal such processes as intensification of ultrasound by sound, nonlinear instability of a sound profile, and prove the validity of the singular focusing condition. Finally, possible applications of the results are outlined. (C) 2001 American Institute of Physics. [DOI: 10.1063/1.1416502]
\end{abstract}

\section{INTRODUCTION}

Liquids with gas bubbles have strongly pronounced nonlinear acoustic properties due to nonlinear oscillations of bubbles and high compressibility of gas. Within recent decades theoretical and experimental investigations have detected many kinds of nonlinear wave phenomena in bubbly fluids, in particular, the ultrasound self-focusing, ${ }^{1,2}$ the sound self-transparency, ${ }^{3}$ wave front conjugation, ${ }^{4}$ the acoustic phase echo, ${ }^{5}$ subharmonic wave generation, ${ }^{6}$ the intensification of sound waves in nonuniform bubbly fluids, ${ }^{7,8}$ the structure formation in acoustic cavitation, ${ }^{9-11}$ and differencefrequency sound generation. ${ }^{12,13}$

To date, long and short waves in bubbly fluids were studied independently. The Korteveg-de Vries (KdV) equation was proposed ${ }^{14}$ for describing the evolution of long-wave disturbances in an ideal liquid with adiabatic gas bubbles. The existence of the long-wave KdV soliton was confirmed by many experiments. ${ }^{15,16}$ The nonlinear Schrödinger (NLS) equation was obtained ${ }^{17}$ as an equation for a short-wave modulation in polydisperse bubbly mixtures. The generation of short-wave subharmonics in bubbly liquids was investigated by a means of geometrical acoustics. ${ }^{6}$

Despite different length scales, long and short waves can interact. Benney ${ }^{18}$ was the first to call attention to the possibility of such a wave interaction on the water surface. The interaction comes into particular prominence when the group

\footnotetext{
a) Author to whom correspondence should be addressed. Present address: Department of Mathematics, Virginia Tech, Blacksburg, VA 24061-0123. Electronic mail: damir@math.vt.edu.
}

speed of short waves is equal to the phase speed of the long wave (the long-wave-short-wave resonance ${ }^{19}$ ). The resonance requires a special type of dispersion relation, e.g., consisting of two branches, and therefore, occurs in a limited number of physical systems. Examples of such systems are waves on the water surface ${ }^{20}$ and waves in a collision-free plasma. ${ }^{21}$ We demonstrate here that the resonance condition is also satisfied for pressure waves in a bubbly fluid.

The interaction of long and short waves is described by nonresonant and resonant models. With no damping the nonresonant one-dimensional model consists of the nonlinear Schrödinger equation for the short-wave envelope and an algebraic correlation between the long-wave profile and a square of the envelope modulus. ${ }^{22}$ The resonant model represents the Zakharov equations. ${ }^{21}$ The two-dimensional interaction is governed by the Davey-Stewartson (DS) system of equations. ${ }^{23}$ There are only two integrable forms of the DS equations referred to as DSI and DSII equations. ${ }^{24}$ In the nonintegrable case the solutions of this system can possess a blowup instability. As a result, singular focusing takes place. $^{25,26}$

The paper is devoted to the theoretical description of the interaction between sound and ultrasound waves in viscous bubbly fluids. The nonlinear wave phenomena presented here, such as degeneracy of the interaction, nonlinear instability of sound waves and singular focusing of ultrasound, are novel for bubbly fluid dynamics. The remainder of the paper is structured as follows. In Sec. II the equations of motion for bubbly fluids and the dispersion relation for plane sound waves in these media are considered. The method of multiple scales is described in Sec. III. We demonstrate this 
method on the problem of weakly two-dimensional sound evolution in a bubbly fluid (Sec. IV). The one-dimensional interaction equations are proposed in Sec. V. We analyze the degeneracy of the interaction and propose new equations for the degenerate resonant interaction in Sec. VI. Section VII deals with the two-dimensional interaction. We construct the DS equations and demonstrate that their unique properties as a dromion solution and singular focusing are true of bubbly fluids. The numerical modeling of the one-dimensional resonant interaction, two-dimensional defocusing and singular focusing of ultrasound is conducted in Sec. VIII. We conclude by some words about possible applications of the results in Sec. IX.

\section{BASIC EQUATIONS}

Consider a mixture of a uniform liquid containing spherical gas bubbles. Assume that the liquid is weakly compressible, all bubbles have the same radius, the pressure in the bubbles varies according to the polytropic law. Suppose that the medium is noncollisional, i.e., we do not take into account direct interactions and collisions between bubbles and the processes of bubble breakup, adhesion, and formation. Neglect the effect of external forces as well as the capillary effects. The motion of such a bubbly fluid is described by the equations ${ }^{27-29}$

$$
\begin{aligned}
& \frac{d \rho}{d t}+\rho \operatorname{div} \mathbf{v}=0, \quad \frac{d n}{d t}+n_{b} \operatorname{div} \mathbf{v}=0, \\
& \rho \frac{d \mathbf{v}}{d t}+\operatorname{grad} p=0 \\
& \rho_{l}\left[a \frac{d^{2} a}{d t^{2}}+\frac{3}{2}\left(\frac{d a}{d t}\right)^{2}\right]+\frac{4 \mu_{e f}}{a} \frac{d a}{d t}=p_{g}-p, \\
& \rho_{l}-\rho_{l 0}=\frac{p-p_{0}}{C_{l}^{2}}, \\
& \rho=\rho_{l}\left(1-\alpha_{g}\right), \quad \alpha_{g}=\frac{4}{3} \pi a^{3} n_{b}, \quad p_{g} / p_{0}=\left(a_{0} / a\right)^{3 \kappa} .
\end{aligned}
$$

Here $\mathbf{v}=u \mathbf{i}+w \mathbf{j}$ is the velocity vector of the mixture; $d / d t$ $=\partial / \partial t+u \partial / \partial x+w \partial / \partial y$ is the substantive derivative with respect to time; $p, \rho$ are the pressure and mass density of the mixture; $\rho_{l}$ is the true density of the liquid; $C_{l}$ is the speed of sound in the pure liquid; $p_{g}, \alpha_{g}, a$, and $n_{b}$ are the pressure, volume content, radius, and number density of the bubbles; $\kappa$ is the polytropic exponent, and the subscript 0 refers to the unperturbed state of the mixture.

The processes in question suggest the oscillatory regime of a radial bubble motion. Then all dissipative effects can take into account on a basis of effective viscosity, ${ }^{29}$ i.e., a certain effective coefficient $\mu_{e f}$, allowing for the liquid viscosity, $\mu_{l}$, thermal damping, $\mu^{(T)}$, and other dissipative mechanisms, is introduced: $\mu_{e f}=\mu_{l}+\mu^{(T)}+\cdots$.

Going over dimensionless variables

$$
\tilde{a}=\frac{a}{a_{0}}-1, \quad \tilde{p}=\frac{p}{p_{0}}-1, \quad \tilde{\rho}=\frac{\rho-\rho_{0}}{\rho_{*}}, \quad \tilde{u}=\frac{u}{v_{*}},
$$

$$
\tilde{w}=\frac{w}{v_{*}}, \quad \tilde{t}=\frac{t}{t_{*}}, \quad \tilde{x}=\frac{x}{l_{*}}, \quad \tilde{y}=\frac{y}{l_{*}},
$$

where

$$
\begin{aligned}
& \rho_{*}=\rho_{0} \alpha_{g 0}, \\
& v_{*}=\left(\frac{\alpha_{g 0} p_{0}}{\rho_{0}}\right)^{1 / 2}, \\
& t_{*}=a_{0}\left(\frac{\rho_{0}}{p_{0}}\right)^{1 / 2}, \\
& l_{*}=\frac{a_{0}}{\left(\alpha_{g 0}\right)^{1 / 2}},
\end{aligned}
$$

and ignoring the terms of order $\alpha_{g 0}$ as compared to unity, one can reduce the system of Eqs. (1) to the following equations for the perturbations in the pressure $\tilde{p}$, mass density $\tilde{\rho}$ and bubble radius $\tilde{a}::^{16,30}$

$\frac{\partial^{2} \rho}{\partial t^{2}}-\frac{\partial^{2} p}{\partial x^{2}}-\frac{\partial^{2} p}{\partial y^{2}}=0$

$\rho-1-b^{2} p+(1+a)^{3}=0$,

$(1+a) \frac{\partial^{2} a}{\partial t^{2}}+\frac{3}{2}\left(\frac{\partial a}{\partial t}\right)^{2}+\frac{\mu^{*}}{1+a} \frac{\partial a}{\partial t}-(1+a)^{-3 \kappa}+p+1=0$,

$b=\left(\frac{p_{0}}{\rho_{l 0} \alpha_{g 0} C_{l}^{2}}\right)^{1 / 2}$,

$\mu^{*}=\frac{4 \mu_{e f}}{a_{0}\left(p_{0} \rho_{0}\right)^{1 / 2}}$

Hereafter the tilde is omitted.

Consider the vector solution to Eqs. (3), $\mathbf{z}=(a, p, \rho)$, in the form of a longitudinal plane harmonic wave propagating along $x: \mathbf{z}=\mathbf{z}_{0} \exp \{i(k x-\omega t)\}$, where $\mathbf{z}_{0}=(A, P, R)$ is the constant vector of the solution amplitudes, $k$ and $\omega$ are the wavenumber and the frequency. The solution is valid when $k$ and $\omega$ are related by the dispersion relation

$$
\omega^{4}+i \mu^{*} \omega^{3}-\left(3 \kappa+\frac{k^{2}+3}{b^{2}}\right) \omega^{2}-\frac{i \mu^{*} k^{2}}{b^{2}} \omega+\frac{3 \kappa k^{2}}{b^{2}}=0 .
$$

Later we shall suppose $k$ and $\omega$ to be non-negative (the wave travels from the left to the right).

In the nondissipative case, $\mu^{*}=0$, relation (5) falls into two branches (Fig. 1)

$$
\begin{aligned}
\omega_{ \pm}^{2}(k)= & \frac{1}{2}\left\{3 \kappa+\left(k^{2}+3\right) b^{-2} \pm\left(\left[3 \kappa-\left(k^{2}+3\right) b^{-2}\right]^{2}\right.\right. \\
& \left.\left.+36 \kappa b^{-2}\right)^{1 / 2}\right\} .
\end{aligned}
$$

The subscripts "-" and "+" identify the lower (or lowfrequency) and upper (or high-frequency) branches, respectively. 


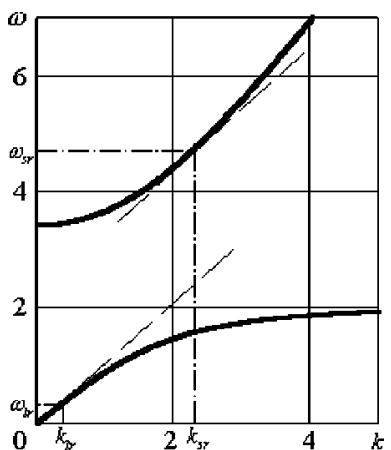

FIG. 1. Dispersion curve for a mixture of water with air bubbles under normal conditions and with a volume gas content $\alpha_{g 0}=1.1 \times 10^{-4}$.

The long-wave asymptotics of the low-frequency branch, $\omega_{l}\left(k_{l}\right)=\left.\omega_{-}\right|_{k \rightarrow 0}$, and the short-wave asymptotics of the high-frequency branch, $\omega_{s}\left(k_{s}\right)=\left.\omega_{+}\right|_{k \rightarrow \infty}$, can be written as follows:

$$
\begin{aligned}
& \omega_{l}=c_{e} k_{l}+O\left(k_{l}^{3}\right) \text { for } k_{l} \rightarrow 0, \\
& \omega_{s}=c_{f} k_{s}+O\left(k_{s}^{-1}\right) \text { for } k_{s} \rightarrow \infty,
\end{aligned}
$$

where

$$
\begin{aligned}
& c_{e}=\omega_{l} /\left.k_{l}\right|_{k_{l} \rightarrow 0}=\left(b^{2}+\kappa^{-1}\right)^{-1 / 2}, \\
& c_{f}=d \omega_{s} /\left.d k_{s}\right|_{k_{s} \rightarrow \infty}=b^{-1}
\end{aligned}
$$

are the equilibrium and frozen speeds of sound in the bubbly mixture.

Dispersion relation (6) allows the existence of the longwave-short-wave resonance. Actually, the group speed of short waves

$$
\begin{aligned}
c_{g}=d \omega_{s} / d k_{s}= & k_{s} \omega_{s}^{-1}\left(\omega_{s}^{2}-3 \kappa\right)\left(2 b^{2} \omega_{s}^{2}-3 \kappa c_{e}^{-2}\right. \\
& \left.-k_{s}^{2}\right)^{-1}
\end{aligned}
$$

is infinitesimal when $k_{s} \rightarrow 0$, and it follows from (2.7) that $c_{f}>c_{e}$. Therefore, for any sufficiently small $k_{l}=k_{l r}$, there exists such a $k_{s}=k_{s r}$ that the long-wave-short-wave resonance condition

$$
c_{g}\left(k_{s r}\right)=c_{p}\left(k_{l r}\right),
$$

is satisfied (the long-dash lines in Fig. 1). Here $c_{p}\left(k_{l}\right)$ $=\omega_{l} / k_{l}$ is the phase speed of the long wave.

The wave numbers of the long and short waves can be related by the formula $k_{l}=\varepsilon_{k} k_{s}$, where $\varepsilon_{k}$ is a certain small parameter. Upon neglecting the terms of order $\varepsilon_{k}$, the resonance condition (9) is simplified

$$
c_{g}\left(k_{s r}\right)=c_{e} .
$$

It should be noted that the long-wave-short-wave resonance in bubbly fluids has nothing to do with the resonance of bubble oscillation which occurs under the condition $\omega$ $=\sqrt{3 \kappa}$ (for reasonably large bubbles). The value $\sqrt{3 \kappa}$ corresponds to the horizontal asymptote of the low-frequency branch. Because the short-wave frequency $\omega_{s}$ belongs to the high-frequency branch, it is always above $\sqrt{3 \kappa}$. The Minnaert resonance is, therefore, beyond the scope of this paper.
Suppose that $\varepsilon_{k}=10^{-3}$ and consider water with air bubbles of the radius $a_{0}=0.1 \mathrm{~mm}$ under normal conditions $\left(p_{0}=0.1 \mathrm{MPa}, \rho_{l 0}=10^{3} \mathrm{~kg} \mathrm{~m}^{-3}\right)$ and with the volume gas content $\alpha_{g 0}=2.22 \times 10^{-4}$. The wavelengths of the short and long waves are then $\lambda_{s} \approx 0.67 \mathrm{~cm}$ and $\lambda_{l} \approx 6.7 \mathrm{~m}$, respectively. The equilibrium speed of sound $C_{e} \equiv c_{e} l_{*} / t_{*}$ $\approx 730 \mathrm{~m} \mathrm{~s}^{-1}$. Thus, the frequency of the long wave $\Omega_{l}$ $=C_{e} / \lambda_{l} \approx 110 \mathrm{~Hz}$ (audible sound). Obviously, the shortwave frequency $\Omega_{s}=\omega_{s} /\left(2 \pi t_{*}\right) \geqslant 1 /\left(2 \pi b t_{*}\right) \approx 35.6 \mathrm{kHz}$ lies in the ultrasound region. Moreover, from (2c) it follows that a decrease in the bubble radius $a_{0}$ leads to increasing the short-wave frequency $\Omega_{s}$. Hence, the long-wave-shortwave interaction in a bubbly fluid can be considered as the interaction of ultrasound and audible sound propagating in this medium.

\section{THE METHOD OF MULTIPLE SCALES}

Let us introduce a parameter $\varepsilon \ll 1$ to satisfy the conditions

$$
\begin{array}{r}
p_{l}=\varepsilon^{l} L, \quad p_{s}=\varepsilon^{s} S, \quad p=p_{l}+p_{s} \exp \{i \Theta\}, \\
L, S=O(1) .
\end{array}
$$

Here $p_{l}, p_{s}$ are the long- and short-wave pressure perturbations in the mixture; $l, s$ are some numbers (exponents of smallness); $\Theta=k_{s} x_{0}-\omega_{s} t_{0}$ is the phase of the short wave. We express similarly the perturbations in the bubble radius and mass density of the mixture. Suppose also that $\varepsilon_{k} \leqslant \varepsilon$.

The nonlinear wave equations describing the interaction between long and short pressure waves may be obtained using the method of multiple scales. ${ }^{31}$ According to this method, the vector solution $\mathbf{z}$ to system (3) expands in powers of the parameter $\varepsilon$ determined above into the long- and short-wave components

$\mathbf{z}=\varepsilon^{l} \sum_{m \geqslant 1} \varepsilon^{m-1} \mathbf{z}_{m}^{(0)}+\sum_{m, n \geqslant 1} \sum \varepsilon^{(s+m-1) n}\left[\mathbf{z}_{m}^{(n)} e^{i n \Theta}+\right.$ c.c. $]$,

and fast $\left(t_{0}, x_{0}, y_{0}\right)$ and slow variables $\left(t_{n}, x_{n}, y_{n}\right)$ $=\varepsilon^{n}\left(t_{0}, x_{0}, y_{0}\right)$, where $n=1,2, \ldots$, are introduced. The introduction of the slow variables results in the following asymptotic series:

$$
\frac{\partial}{\partial \psi} \rightarrow \frac{\partial}{\partial \psi_{0}}+\sum_{n \geqslant 1} \varepsilon^{n} \frac{\partial}{\partial \psi_{n}}, \quad \psi=t, x, \text { or } y .
$$

The long- and short-wave components $\mathbf{z}_{m}^{(0)}, \mathbf{z}_{m}^{(n)}, m, n$ $=1,2, \ldots$ depend only on the slow variables. The short wave is considered to be plane because the phase $\Theta$ does not contain the fast variable $y_{0}$, but its amplitude may depend on the slow variables $y_{n}, n=1,2, \ldots$. The numbers $(l, s)$ play a key role in this asymptotic method because they define a type of long-wave-short-wave interaction equations. ${ }^{30}$

We make use of the following "multiscale-expansion" procedure for the derivation of interaction equations:

(1) Once we have decided upon the values of $l$ and $s$, we substitute the multiscale expansions (12) and (13) into the equations for perturbations (3). In doing so, we restrict our attention to cubic terms of Eqs. (3), because the 
higher-order nonlinearities have negligibly small effects on the long-wave-short-wave interaction. ${ }^{32}$

(2) The resulting system of equations is split into the zeroth $(n=0)$, first $(n=1)$, and second harmonics $(n=2)$ and then, if possible, reduces to one equation for each harmonic of pressure perturbation $p$.

(3) The algebraic relationship between the second-harmonic amplitude $p_{1}^{(2)}$ and a square of the first-harmonic amplitude $\left[p_{1}^{(1)}\right]^{2}$ is deduced from the leading order of the second-harmonic equation.

(4) Using this relationship, we eliminate $p_{1}^{(2)}$ from the firstharmonic equation and obtain the coupled system of the zeroth- and first-harmonic equations. The system is not closed yet. We should also eliminate the unknowns $p_{m}^{(0)}$ and $p_{m}^{(1)}$, where $m=2,3, \ldots$, from the equations. To do this would require some assumptions of the unknowns. The easiest way is to assume that they are equal to zero.

(5) Now, each of the obtained equations contains the terms of different orders. The next step is therefore splitting the equations into the orders of $\varepsilon$. With a few exceptions (see below), we follow this. The expressions appearing in each order of $\varepsilon$ are equated to zero. This gives a bunch of asymptotic equations for the long-wave profile $p_{1}^{(0)}$ and short-wave envelope $p_{1}^{(1)}$. Among the equations involving the interaction terms, the lowermost-order ones represent the equations for long-wave-short-wave interaction. In particular, the interaction terms of the lowermost order for the short and long waves are $p_{1}^{(0)} p_{1}^{(1)}$ and the second derivative of $\left|p_{1}^{(1)}\right|^{2}$ (with respect to $t$ or $x$ ), respectively. They come from quadratic nonlinearity. From (12) and (13), it follows that the interaction equations occur in the orders of $\varepsilon^{l+s}$ (for the short wave) and $\varepsilon^{2 s+2}$ (for the long wave). The lowerorder equations, when present, are linear and without dispersive terms. (This can be arranged by choosing the numbers $l$ and $s$.) They give additional information about interacting waves: the waves interact in the frame moving with the group speed of short waves and so on. The issue, which can not be resolved by choosing $l$ and $s$, emerges in the case of degeneracy, when the above interaction terms vanish. In the degeneracy case, interaction is of higher order of $\varepsilon$ than dispersion. We cannot cut down the dispersive terms and should take into account the interaction terms. The equations for degenerate interaction therefore contain the terms of different orders of $\varepsilon$.

\section{WEAKLY TWO-DIMENSIONAL EVOLUTION OF SOUND WAVES}

Consider the method of multiple scales on the problem of weakly two-dimensional evolution of sound waves in bubbly fluids. Substitute the multiscale expansions (12) and (13) into Eqs. (3) provided that there is no ultrasound $\left(\mathbf{z}_{m}^{(n)}=0\right.$ and $l=2$, where $n=1,2, \ldots)$ and the derivatives of $\mathbf{z}_{m}^{(0)}$ with respect to $y$ are much less than those with respect to $x$, i.e., all unknowns do not depend on $y_{1}$. This means that all changes in $y$ are slower than in $x$ (in the direction of wave motion) and can be considered as transverse perturbations in the wave profile. Then the following equations (of order $\varepsilon^{4}$ )

$$
\begin{aligned}
& {\left[\kappa\left(1-b^{2} c^{2}\right)-1\right] \frac{\partial^{2} a_{1}^{(0)}}{\partial \xi^{2}}=0,} \\
& \rho_{1}^{(0)}=-3\left(1+\kappa b^{2}\right) a_{1}^{(0)}, \\
& p_{1}^{(0)}=-3 \kappa a_{1}^{(0)}
\end{aligned}
$$

will be true. Here $\xi=x_{1}-c t_{1}$. Equations (14) have a unique solution when $c=c_{e}$ [cf. (7a)]. Thus, long waves travel with the equilibrium speed of sound $C_{e}=c_{e} l_{*} / t_{*}$ in bubbly fluids. In view of (14) and $c=c_{e}$, the Kadomtsev-Petviashvili (KP) equation ${ }^{33}$ for the sound profile $L=p_{1}^{(0)}$ occurs in the order of $\varepsilon^{6}$

$$
\frac{\partial}{\partial \xi}\left(\frac{\partial L}{\partial \tau}+2 \sigma L \frac{\partial L}{\partial \xi}+\chi \frac{\partial^{3} L}{\partial \xi^{3}}\right)+\frac{1}{2} \frac{\partial^{2} L}{\partial \zeta^{2}}=0 .
$$

Here $\tau=t_{2}$ and $\zeta=y_{2}$. The coefficients $\sigma=(\kappa$ $+1) c_{e}^{3} /\left(4 \kappa^{2}\right)$ and $\chi=c_{e}^{5} /\left(6 \kappa^{2}\right)$ are always positive. When denoting

$$
T=\tau \sqrt{\frac{\sigma^{3}}{27 \chi}}, \quad X=\xi \sqrt{\frac{\sigma}{3 \chi}}, \quad Y=\zeta \sqrt{\frac{\sigma^{3}}{162 \chi}},
$$

Eq. (15) takes the canonical form

$$
\frac{\partial}{\partial X}\left(\frac{\partial L}{\partial T}+6 L \frac{\partial L}{\partial X}+\frac{\partial^{3} L}{\partial X^{3}}\right)+3 \sigma_{\mathrm{KP}} \frac{\partial^{2} L}{\partial Y^{2}}=0
$$

with $\sigma_{\mathrm{KP}}=1$, which is referred to as KPI equation. ${ }^{34}$ The sign of $\sigma_{\mathrm{KP}}$ may be negative for a number of other physical systems (nonlinear optics and water waves ${ }^{25}$ ) and Eq. (17) is then known as KPII equation. Both the KPI and KPII are integrable by means of the inverse scattering transform. ${ }^{34-36}$ The simplest soliton solution to (17), regardless of $\sigma_{\mathrm{KP}}$, is given by

$$
L=2 V^{2} \operatorname{sech}^{2} V\left(X-4 V^{2} T-X_{0}\right), \quad V, X_{0}=\text { const, }
$$

which represents the famous $\mathrm{KdV}$ soliton. This is not surprising, since the KdV equation follows from (17) in the case that $L$ does not depend on $\zeta$ (the one-dimensional sound wave):

$$
\frac{\partial L}{\partial T}+6 L \frac{\partial L}{\partial X}+\frac{\partial^{3} L}{\partial X^{3}}=0 .
$$

The important question arises of whether such a solitary wave is unstable in the bubbly mixture with respect to transverse perturbations, i.e., whether these perturbations destroy the one-dimensional solution (18). There are a number of analytical $^{25,33,37,38}$ and numerical works ${ }^{39,40}$ which showed that if $\sigma_{\mathrm{KP}}=-1$ (the KPII case), a $\mathrm{KdV}$ soliton is linearly unstable: It transforms into a chain of two-dimensional line solitons and/or lumps under a periodic transverse perturbation. In contrast, a numerical simulation using the KPI equation, ${ }^{41}$ which holds for bubbly mixtures as shown above, reveals that the solution (18) is stable. Hence, there is no transverse instability of solitary waves in bubbly liquids. Previously, Gavrilyuk has argued that the KPII equation is valid 
for bubbly liquids, and therefore, "solitary waves in bubbly liquids are linearly unstable." 42 However, if these waves were unstable with respect to transverse perturbations, the experimental results of Nakoryakov and his co-workers ${ }^{14}$ on the existence of $\mathrm{KdV}$ solitons in bubbly liquids would be questionable. This is because such experiments would call for using extremely narrow channels wherein the dependence of the wave profile on the transverse coordinate could be neglected. Fortunately, Gavriluk's conclusion was in error.

\section{ONE-DIMENSIONAL INTERACTION}

Let us put $(l, s)=(2,1)$ and substitute expansions (12) and (13) into Eqs. (3). Suppose that all unknowns do not depend on $y_{0}, y_{1}, y_{2}, \ldots$ (the one-dimensional case). We then split the resulting expressions into harmonics of the short wave, $\exp \{i n \Theta\}, n=0,1,2, \ldots$ and restrict our attention to order $\varepsilon^{4}$ for the zeroth harmonic and $\varepsilon^{3}$ for the first harmonic. We also assume that the damping coefficient $\mu^{*}$ is of order $\varepsilon^{2}$ (weak damping): $\mu^{*}=\varepsilon^{2} \mu, \mu \sim O(1)$. Then, the following equations for interaction will be true:

$$
\begin{aligned}
& L=L_{0}+\frac{\alpha}{c_{g}^{2}-c_{e}^{2}} \frac{\partial^{2}|S|^{2}}{\partial \xi^{2}}, \\
& i \frac{\partial S}{\partial \tau}+\beta \frac{\partial^{2} S}{\partial \xi^{2}}+i \Gamma S+\gamma|S|^{2} S=\delta L S .
\end{aligned}
$$

Here $L=p_{1}^{(0)}$ is the sound profile; $S=p_{1}^{(1)}$ is the ultrasound envelope. In doing so, $L=L(\xi)+L_{0}, S=S(\tau, \xi), \tau=t_{2}, \xi$ $=x_{1}-c_{g} t_{1}\left(L_{0}\right.$ is the initial sound profile which is assumed to be constant). The coefficients

$$
\begin{aligned}
\alpha= & -\frac{c_{e}^{2} c_{g}^{2}}{\kappa\left(\omega_{s}^{2}-3 \kappa\right)^{2}}\left[\omega_{s}^{2}-9 \kappa(\kappa+1)\right] \\
\beta= & \frac{c_{g}}{2 k_{s}}\left[1+\frac{c_{g}}{\omega_{s}^{2}-3 \kappa}\left\{4 k_{s} \omega_{s}+c_{g}\left(k_{s}^{2}+3\left(1+\kappa b^{2}\right)\right.\right.\right. \\
& \left.\left.\left.-6 b^{2} \omega_{s}^{2}\right)\right\}\right] \\
\delta= & \frac{c_{g} \omega_{s}^{2}}{2 \kappa k_{s}\left(\omega_{s}^{2}-3 \kappa\right)^{2}}\left[\omega_{s}^{2}-9 \kappa(\kappa+1)\right] \\
\Gamma= & \frac{\mu \omega_{s} c_{g}\left(b^{2} \omega_{s}^{2}-k_{s}^{2}\right)}{2 k_{s}\left(\omega_{s}^{2}-3 \kappa\right)}, \\
\gamma= & -\frac{\left[\omega_{s}^{2}-3 \kappa(3 \kappa+1)\right] \delta}{\left(\omega_{s}^{2}-3 \kappa\right)^{2}}-\frac{c_{g}\left(b^{2} \omega_{s}^{2}-k_{s}^{2}\right)}{4 k_{s} \omega_{s}^{2}\left(\omega_{s}^{2}-3 \kappa\right)^{3}}\left[-\omega_{s}^{4}\right. \\
& \left.+27 \kappa^{2}(\kappa+1) \omega_{s}^{2}+27 \kappa^{2}(\kappa+1)^{2}\right] .
\end{aligned}
$$

$c_{g}$ and $c_{e}$ are determined from (8) and (7a).

Due to the absence of derivatives in (20a) the resulting model describes the formation of an inertialess sound by an ultrasound wave packet and their subsequent interaction.
Substitution (20a) into (20b) and notation $\widetilde{S}=S \exp \left\{i \delta L_{0} \tau\right\}$ give the Ginzburg-Landau (GL) equation in $\widetilde{S}$

$$
i \frac{\partial \widetilde{S}}{\partial \tau}+\beta \frac{\partial^{2} \widetilde{S}}{\partial \xi^{2}}+\gamma^{\prime}|\widetilde{S}|^{2} \widetilde{S}+i \Gamma \widetilde{S}=0, \quad \gamma^{\prime}=\gamma-\frac{\alpha \delta}{c_{g}^{2}-c_{e}^{2}}
$$

Generally, the GL equation does not have analytical solutions. ${ }^{32}$ But in the nondissipative case $(\Gamma=0)$, Eq. (22) known as the nonlinear Schrödinger (NLS) equation is integrable. The NLS equation possesses soliton solutions ${ }^{43}$ if the Benjamin-Feir instability criterion ${ }^{44}$

$$
\beta \gamma^{\prime}>0
$$

is satisfied. It is worth noting that the Benjamin-Feir instability is the modulational (temporal) instability of spatially uniform solutions to the nonlinear Schrödinger equation. The unstable spatially uniform solution is deformed into a train of localized waves called bright NLS solitons. ${ }^{38}$ For bubbly fluids, the Benjamin-Feir instability regions were given on the parameter plane $\left(k_{s}, b\right)$ earlier. ${ }^{16}$ The physical significance of this instability in the bubbly fluids may be the following. Let us assume that a monochromatic ultrasound wave propagates through the bubbly medium. Its amplitude $S$ does not depend on $x$ and, therefore, represents the spatially uniform solution to the NLS equation. Suppose that the condition (23) is satisfied for the wave, i.e., the values for the wave frequency and bubbly fluid parameters are in the Benjamin-Feir instability region. This monochromatic wave will be unstable to spatial periodic perturbations. As a result, a train of localized short-wave packets will be generated with the passage of time. Hence, it is impossible to sustain a monochromatic ultrasonic signal in the bubbly fluid if the Benjamin-Feir instability criterion is satisfied (spatial pressure perturbations inevitably occur in experiments).

The interaction model (20) becomes incorrect if $c_{g}=c_{e}$ (the resonant case). This is because the term on the righthand side of (20a) goes to infinity. It is necessary to change the exponents of smallness $l$ and $s$. Under resonance condition $(10)$ and $(l, s)=(2,3 / 2)$, substitution of expansions (12) and (13) into system (3) results in the Zakharov equations with damping (which arises in the orders of $\varepsilon^{5}$ for the zeroth harmonic and $\varepsilon^{7 / 2}$ for the first harmonic):

$$
\frac{\partial L}{\partial \tau}+\frac{\alpha}{2 c_{e}} \frac{\partial|S|^{2}}{\partial \xi}=0, \quad i \frac{\partial S}{\partial \tau}+\beta \frac{\partial^{2} S}{\partial \xi^{2}}+i \Gamma S=\delta L S .
$$

Now $L=L(\tau, \xi), S=S(\tau, \xi), \tau=t_{2}, \xi=x_{1}-c_{g} t_{1}$.

In contrast to the previous (nonresonant) model, the effects of sound on ultrasound are nontrivial. The dynamics of resonant sound-ultrasound interaction depends on the initial conditions for the sound wave.

The Zakharov equations, at $\Gamma=0$, is one of the integrable nonlinear wave models. Their spatially uniform solutions are always unstable. The instability evolves for the perturbations with the wave number $K<\left(6 \sqrt{3}|\alpha \delta|\left|S_{0}\right|^{2}\right)^{1 / 3}$. As a result, there exist the envelope soliton solutions ${ }^{45}$ for any values of the coefficients. 


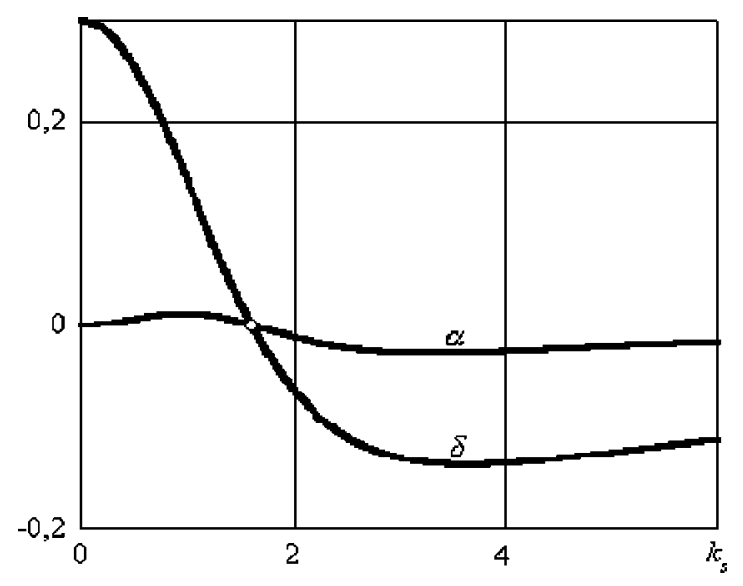

FIG. 2. Coefficients $\alpha$ and $\delta$ versus the wave number $k_{s}(\kappa=1.4, b$ $=0.447)$. The white point corresponds to the degeneracy of the interaction.

\section{DEGENERACY OF THE INTERACTION}

The NLS and Zakharov equations are classical models for the long-wave-short-wave interaction. Are there any special features of the sound-ultrasound interaction in bubbly fluids? It turns out there exists one peculiarity.

In the bubbly fluids, the interaction coefficients $\alpha$ and $\delta$ can vanish simultaneously (Fig. 2). This happens when the ultrasound frequency

$$
\omega_{s}=3[\kappa(\kappa+1)]^{1 / 2} .
$$

The interaction between sound and ultrasound is then degenerated because the equations for interaction are separated.

However, such a degeneracy does not mean the absence of interaction. Certainly, the sound profile $L(\xi, \zeta)$ is equal to zero in the nonresonant degenerate case. But if we take into account the zeroth-harmonic terms up to order $\varepsilon^{5}$, the interaction between $S=p_{1}^{(1)}$ and $L_{1}=p_{2}^{(0)}$ can occur

$$
\begin{aligned}
& L_{1}=\frac{i \lambda}{c_{g}^{2}-c_{e}^{2}}\left[S \frac{\partial S^{*}}{\partial \xi}-S^{*} \frac{\partial S}{\partial \xi}\right], \\
& i \frac{\partial S}{\partial \tau}+\beta \frac{\partial^{2} S}{\partial \xi^{2}}+i \Gamma S+\gamma|S|^{2} S=0 .
\end{aligned}
$$

The coefficient $\lambda=(\kappa+1)^{1 / 2} c_{e}^{2} c_{g}^{3} /\left[3 \kappa^{5 / 2}(3 \kappa+2)^{2}\right]$ never vanishes. Here the quasimonochromatic ultrasonic signal will also generate sound but of much smaller intensity than in the nondegenerate case.

\section{A. Resonant degeneracy: The case $(I, s)=(2,3 / 2)$}

Under condition (25) Eqs. (24) turn into the uncoupled equations:

$$
\begin{aligned}
& \frac{\partial L}{\partial \tau}=0, \\
& i \frac{\partial S}{\partial \tau}+\beta \frac{\partial^{2} S}{\partial \xi^{2}}+i \Gamma S=0 .
\end{aligned}
$$

From (27a) it follows the invariance of an initial sound profile with respect to the time $\tau$. Equation (27b) describes the linear dispersion and dissipation of the ultrasound wave packet.

To construct the model of the degenerate resonant interaction we substitute (12) and (13) into (3) and consider all terms up to order $\varepsilon^{6}$ for the long-wave component and $\varepsilon^{9 / 2}$ for the short-wave one. Again $L=p_{1}^{(0)}$ and $S=p_{1}^{(1)}$ are functions of $\xi=x_{1}-c_{g} t_{1}, \tau=t_{2}$. Denote other components as $L_{1}=p_{2}^{(0)}, L_{2}=p_{3}^{(0)}, S_{1}=p_{2}^{(1)}, S_{2}=p_{3}^{(1)}$ and assume that $L_{1}$ and $S_{1}$ evolve in accordance with Eqs. (27) and $L_{2}$ $=L_{2}(\xi), S_{2}=S_{2}(\xi)$. Then, under conditions (25) and (10), the degenerate sound-ultrasound interaction is governed by the equations

$$
\begin{aligned}
& \frac{\partial L}{\partial \tau}+\varepsilon\left\{\chi \frac{\partial^{3} L}{\partial \xi^{3}}+\sigma \frac{\partial L^{2}}{\partial \xi}-i \lambda\left[S^{*} \frac{\partial^{2} S}{\partial \xi^{2}}-S \frac{\partial^{2} S^{*}}{\partial \xi^{2}}\right]\right\}=0, \\
& \frac{\partial S}{\partial \tau}-i \beta \frac{\partial^{2} S}{\partial \xi^{2}}+\Gamma S+\varepsilon\left\{\eta \frac{\partial^{3} S}{\partial \xi^{3}}+i \gamma S^{2} S^{*}+\Gamma^{\prime} \frac{\partial S}{\partial \xi}-\nu L \frac{\partial S}{\partial \xi}\right. \\
& \left.-\frac{\nu}{2} S \frac{\partial L}{\partial \xi}\right\}=0 .
\end{aligned}
$$

Equations (28) describe the degenerate interaction between low-intensity sound and ultrasound. This is because the amplitude of the sound wave is less than the ultrasound envelope $(l>s)$. Obviously, if $S=0$, Eq. (28b) reduces to the $\mathrm{KdV}$ equation (19) in a more slower time $t_{3}=\varepsilon t_{2}$.

It should be noted that (28) is determined only by the parameter $\kappa$. First, this follows from the degeneracy condition (25) according to which the ultrasound frequency $\omega_{s r}$ depends only on $\kappa$. Second, when (25) is substituted into the dispersion relation (6), a relation is obtained which relates the quantities $k_{s r}, b$, and $\kappa$. Together with the resonance condition (10) the latter expression constitutes a set of two algebraic equations in $k_{s r}$ and $b$ with the parameter $\kappa$. Hence $k_{s r}, b$ are completely expressed via $\kappa$

$$
\begin{aligned}
& \omega_{s r}=3[\kappa(\kappa+1)]^{1 / 2}, \quad k_{s r}=\frac{3(\kappa+1)}{\kappa^{1 / 2}(3 \kappa+2)}, \\
& b=\frac{[3 \kappa(\kappa+1)+1]^{1 / 2}}{\kappa(3 \kappa+2)} .
\end{aligned}
$$

For example, if one considers a mixture of water with air bubbles of the radius $a_{0}=0.1 \mathrm{~mm}$, under normal conditions and the volume gas content $\alpha_{g 0} \approx 3 \cdot 10^{-4}$, the degeneracy of resonant interaction occurs when the ultrasound frequency $f_{s r} \approx 87.5 \mathrm{kHz}$.

All coefficients of system (28) are positive at $\kappa \geqslant 1$

$$
\begin{gathered}
c_{g}=c_{e}=\frac{\kappa(3 \kappa+2)}{(3 \kappa+1)(\kappa+1)^{1 / 2}}, \\
\sigma=\frac{\kappa(3 \kappa+2)^{3}}{4(3 \kappa+1)^{3}(\kappa+1)^{1 / 2}},
\end{gathered}
$$




$$
\begin{aligned}
\lambda= & \frac{\kappa^{3 / 2}}{6(3 \kappa+1)^{4}(3 \kappa+2)(\kappa+1)^{3 / 2}}\left[27 \kappa^{3}+18 \kappa^{2}+18 \kappa\right. \\
& +4] \\
\beta= & \frac{3 \kappa^{5 / 2}(3 \kappa+2)}{2(\kappa+1)^{3 / 2}(3 \kappa+1)^{3}}\left[3 \kappa^{2}+3 \kappa+2\right] \\
\eta= & \frac{\kappa^{3}(3 \kappa+2)}{6(3 \kappa+1)^{5}(\kappa+1)^{5 / 2}}\left[81 \kappa^{4}+54 \kappa^{3}+81 \kappa^{2}+60 \kappa\right. \\
& +28], \\
\gamma= & \frac{(\kappa+1)^{1 / 2}}{12 \kappa^{1 / 2}(3 \kappa+1)(3 \kappa+2)^{2}}[9 \kappa-2] \\
\chi= & \frac{\kappa^{3}(3 \kappa+2)^{5}}{6(\kappa+1)^{5 / 2}(3 \kappa+1)^{5}}, \quad \Gamma=\frac{3 \kappa \mu}{2(3 \kappa+1)} \\
\nu= & \frac{\kappa(3 \kappa+2)}{(3 \kappa+1)^{2}(\kappa+1)^{1 / 2}}, \quad \kappa^{3 / 2} \mu \\
& \left.+288 \kappa^{2}+79 \kappa+4\right] \\
& \frac{30}{2(\kappa+1)^{2}(3 \kappa+1)^{3}(3 \kappa+2)} \\
& \\
& \\
&
\end{aligned}
$$

Due to the presence of the damping term $\Gamma S$ of lower order $\varepsilon$ in comparison with the interaction terms, the fast decay of the ultrasonic signal takes place. This leads to the negligible action of ultrasound on sound. In order to "catch" effects of sound on ultrasound, we need large sound amplitudes. But this seems to contradict the perturbation theory according to which the sound profile $L$ should be less than $\varepsilon^{-1}$. Consequently, we may argue that weak damping suppresses the resonant interaction between low-intensity sound and ultrasound in the case of degeneracy.

\section{B. Resonant degeneracy: The case $(I, s)=(1,1)$}

It turns out that the resonant degenerate interaction can be described by other models. One of such models occurs when $(l, s)=(1,1)$, i.e., in the context of high-intensity sound in the ultrasonic field. trivial

With choosing $(l, s)=(1,1)$, the interaction is usually

$$
L=L_{0}(\xi), \quad i \frac{\partial S}{\partial t_{1}}=\delta L S
$$

But provided that the conditions of resonant degeneracy (29) are satisfied, i.e., $\delta=0$, and damping is weak, the following equations (of order $\varepsilon^{3}$ for $L$ and $S$ ) will govern the interaction in the time $\tau=t_{2}$ :

$$
\begin{aligned}
\frac{\partial L}{\partial \tau}+ & \sigma \frac{\partial L^{2}}{\partial \xi}+\varepsilon\left\{\chi \frac{\partial^{3} L}{\partial \xi^{3}}+\varsigma \frac{\partial L^{3}}{\partial \xi}+\varrho \frac{\partial L|S|^{2}}{\partial \xi}+\widetilde{\Gamma}|S|^{2}\right. \\
& \left.-i \lambda\left(S^{*} \frac{\partial^{2} S}{\partial \xi^{2}}-S \frac{\partial^{2} S^{*}}{\partial \xi^{2}}\right)\right\}=0,
\end{aligned}
$$

$$
i \frac{\partial S}{\partial \tau}+\beta \frac{\partial^{2} S}{\partial \xi^{2}}-\gamma S^{2} S^{*}+i \Gamma S=i \nu L \frac{\partial S}{\partial \xi}+i \frac{\nu}{2} S \frac{\partial L}{\partial \xi}+\widetilde{\delta} L^{2} S .
$$

Here

$$
\begin{aligned}
& \widetilde{\Gamma}=\frac{2 \mu}{3 \kappa(3 \kappa+1)(3 \kappa+2)^{2}}, \\
& \widetilde{\delta}=\frac{(3 \kappa+2)^{2}}{36 \kappa^{1 / 2}(\kappa+1)^{1 / 2}(3 \kappa+1)}, \\
& \mathrm{\varsigma}=\frac{(3 \kappa+2)^{3}}{36(\kappa+1)^{3 / 2}(3 \kappa+1)^{5}}\left[27 \kappa^{4}+62 \kappa^{3}+45 \kappa^{2}+7 \kappa-2\right]
\end{aligned}
$$

$\varrho=\frac{1}{6(\kappa+1)^{1 / 2}(3 \kappa+1)^{5}(3 \kappa+2)}\left[27 \kappa^{3}+36 \kappa^{2}-4\right] ;$

the other coefficients were defined above.

We include the terms of order $\varepsilon$ in Eq. (32a). Otherwise, the sound wave will be governed by the Hopf equation

$$
\frac{\partial L}{\partial \tau}+\sigma \frac{\partial L^{2}}{\partial \xi}=0
$$

The Hopf equation describes the process of shock wave formation from an initially smooth profile. Mathematically this implies that the derivatives of $L$ and $S$ with respect to $\xi$ must go to infinity at some spatial point $\xi=\xi_{*}$ in a finite time. Hence, the terms of order $\varepsilon$, containing these derivatives, become rather large to be able to influence the dynamics of sound-ultrasound interaction.

\section{TWO-DIMENSIONAL INTERACTION}

Let us consider the two-dimensional interaction, i.e., let us take into account the dependence of wave perturbations on the transverse coordinate $y$. When $(l, s)$ equals $(2,1)$, substitution of multiscale expansions (12) and (13) into Eqs. (3) results in the Davey-Stewartson (DS) equations with damping

$$
\begin{aligned}
& \left(c_{g}^{2}-c_{e}^{2}\right) \frac{\partial^{2} L}{\partial \xi^{2}}-c_{e}^{2} \frac{\partial^{2} L}{\partial \zeta^{2}}=\alpha \frac{\partial^{2}|S|^{2}}{\partial \xi^{2}}, \\
& i \frac{\partial S}{\partial \tau}+\beta \frac{\partial^{2} S}{\partial \xi^{2}}+\varrho \frac{\partial^{2} S}{\partial \zeta^{2}}+i \Gamma S+\gamma|S|^{2} S=\delta L S .
\end{aligned}
$$

Here $L \equiv p_{1}^{(0)}=L(\xi, \zeta)+L_{0} ; S \equiv p_{1}^{(1)}=S(\tau, \xi, \zeta) ; \tau=t_{2} ; \xi$ $=x_{1}-c_{g} t_{1} ; \zeta=y_{1}$. The coefficient

$$
\varrho=\frac{c_{g}}{2 k_{s}} \text {. }
$$

Unlike the one-dimensional case, the resonance condition $c_{g}=c_{e}$ does not require to change the exponents of smallness $l$ and $s$, and the form of equations is conserved.

It should be noted Eq. (35a) does not contain the term $\partial^{2}|S|^{2} / \partial \zeta^{2}$. This term disappears in going from the radius 
perturbations $a_{1}^{(0)}$ and $a_{1}^{(1)}$ to the pressure perturbations $p_{1}^{(0)}$ and $p_{1}^{(1)}$. In particular, the zeroth-harmonic equation for $a$ has the form

$$
\begin{aligned}
& {\left[\frac{\partial^{2}}{\partial t_{1}^{2}}-c_{e}^{2}\left(\frac{\partial^{2}}{\partial x_{1}^{2}}+\frac{\partial^{2}}{\partial y_{1}^{2}}\right)\right] a_{1}^{(0)}} \\
& =\left[\alpha_{1} \frac{\partial^{2}}{\partial t_{1}^{2}}+\alpha_{2}\left(\frac{\partial^{2}}{\partial x_{1}^{2}}+\frac{\partial^{2}}{\partial y_{1}^{2}}\right)\right]\left|a_{1}^{(1)}\right|^{2},
\end{aligned}
$$

where

$$
\begin{aligned}
& \alpha_{1}=-\frac{6+b^{2}\left[\omega_{s}^{2}-3 \kappa(3 \kappa+1)\right]}{3\left(1+\kappa b^{2}\right)}, \\
& \alpha_{2}=\frac{\omega_{s}^{2}-3 \kappa(3 \kappa+1)}{3\left(1+\kappa b^{2}\right)} .
\end{aligned}
$$

From Eq. (3) it follows that

$$
\begin{aligned}
& a_{1}^{(1)}=\frac{p_{1}^{(1)}}{\left(\omega_{s}^{2}-3 \kappa\right)}+O(\varepsilon), \\
& a_{1}^{(0)}=-\frac{1}{3 \kappa}\left[p_{1}^{(0)}+\frac{3 \alpha_{2}\left(1+\kappa b^{2}\right)}{\left(\omega_{s}^{2}-3 \kappa\right)^{2}}\left|p_{1}^{(1)}\right|^{2}\right]+O(\varepsilon) .
\end{aligned}
$$

Substitution of Eqs. (39) into Eq. (38) leads to the cancellation of the second derivatives of $\left|p_{1}^{(1)}\right|^{2}$ with respect to $x_{1}$ and $y_{1}$. We then have the following zeroth-harmonic equation for $p$ :

$$
\frac{\partial^{2} p_{1}^{(0)}}{\partial t_{1}^{2}}-c_{e}^{2}\left(\frac{\partial^{2}}{\partial x_{1}^{2}}+\frac{\partial^{2}}{\partial y_{1}^{2}}\right) p_{1}^{(0)}=\frac{\alpha}{c_{g}^{2}} \frac{\partial^{2}\left|p_{1}^{(1)}\right|^{2}}{\partial t_{1}^{2}},
$$

which is reduced to Eq. (35a).

\section{A. Dromions in bubbly fluids}

When $\delta \neq 0$ and $\Gamma=0$, Eqs. (35) can be transformed into the canonical form. ${ }^{20,23}$ For this purpose, we perform the substitutions $L(\xi, \zeta) \rightarrow \delta^{-1} \partial \Psi / \partial \xi$ and $\widetilde{S}=S \exp \left\{i \delta L_{0} \tau\right\}$ and introduce the notation $\sigma=c_{e}^{-2}\left(c_{e}^{2}-c_{g}^{2}\right)$ :

$$
\begin{aligned}
& \sigma \frac{\partial^{2} \Psi}{\partial \xi^{2}}+\frac{\partial^{2} \Psi}{\partial \zeta^{2}}=-\frac{\alpha \delta}{c_{e}^{2}} \frac{\partial|\widetilde{S}|^{2}}{\partial \xi}, \\
& i \frac{\partial \widetilde{S}}{\partial \tau}+\beta \frac{\partial^{2} \widetilde{S}}{\partial \xi^{2}}+\varrho \frac{\partial^{2} \widetilde{S}}{\partial \zeta^{2}}+\gamma|\widetilde{S}|^{2} \widetilde{S}=\widetilde{S} \frac{\partial \Psi}{\partial \xi} .
\end{aligned}
$$

It is known ${ }^{24}$ that Eqs. (41) are nonintegrable for most values of the coefficients. The exception is the case when (41) can be represented as

$$
\begin{aligned}
& \delta_{1} \frac{\partial^{2} Q}{\partial x^{2}}+\frac{\partial^{2} Q}{\partial y^{2}}=-\delta_{2} \frac{\partial|A|^{2}}{\partial x}, \\
& i \frac{\partial A}{\partial t}+\nu \frac{\partial^{2} A}{\partial x^{2}}+\frac{\partial^{2} A}{\partial y^{2}}=-\nu|A|^{2} A+A \frac{\partial Q}{\partial x},
\end{aligned}
$$

with $\nu= \pm 1, \delta_{1}=-\nu, \delta_{2}=2$.

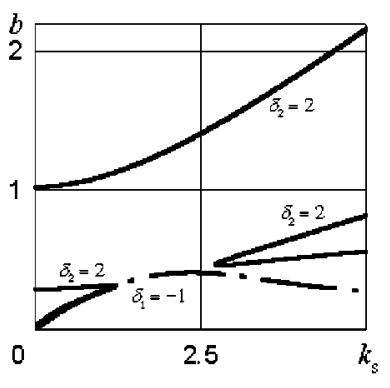

(a)

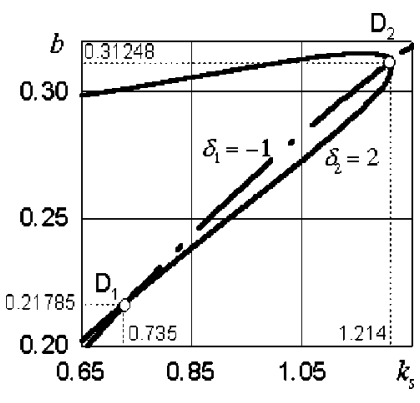

(b)
FIG. 3. Map of parameter plane, showing where the DSI equations govern the sound-ultrasound interaction in bubbly fluids.

Depending on the sign of $\nu$, Eqs. (42) are divided into the Davey-Stewartson I (DSI) equations, of hyperbolicelliptic type $(\nu=1)$, and the Davey-Stewartson II (DSII) equations, of elliptic-hyperbolic type $(\nu=-1)$. The integrability of the DSI and DSII equations by inverse scattering transform was proved in numerous publications (the reader is referred to Ref. 35 for a list of relevant articles).

Since the coefficients $\beta$ and $\varrho$ in (35) are always positive [cf. (21) and (36)], the DSI case is only possible for the interacting waves in bubbly fluids. To show this, it is enough to perform the substitutions $Q=\Psi, A=\gamma^{1 / 2} \widetilde{S}, x=\xi / \beta^{1 / 2}, y$ $=\zeta / \varrho^{1 / 2}, t=\tau$ in Eqs. (41). The DSI equations occur when

$$
\delta_{1}=\frac{\varrho}{\beta}\left[1-c_{g}^{2} / c_{e}^{2}\right]=-1, \quad \delta_{2}=\frac{\alpha \delta \varrho}{\gamma \beta^{1 / 2} c_{e}^{2}}=2 .
$$

You might see before that the coefficients $\alpha$ and $\delta$ were of the same sign (Fig. 2). Hence, to satisfy the first condition of (43), $\gamma$ should be positive. Then the radicand in the second condition is also positive.

The intersection of the curves $\delta_{1}=-1$ and $\delta_{2}=2$ on the parameter plane $\left(k_{s}, b\right)$, plotted in Fig. 3(a), proves the satisfaction of conditions (43). Figure 3(b) shows two points of this intersection $\left(D_{1}\right.$ and $\left.D_{2}\right)$ that correspond to the volume gas contents $\alpha_{g 01} \approx 2.04 \times 10^{-4}, \alpha_{g 02} \approx 1.42 \times 10^{-4}$ and the ultrasound frequencies $\Omega_{s 1} \approx 0.11 \mathrm{MHz}, \Omega_{s 2} \approx 0.14 \mathrm{MHz}$ (in the case of a mixture of water with air bubbles of the radius $0.1 \mathrm{~mm}$ under normal conditions).

Among all solutions of the DSI equation, so-called "dromion" solution ${ }^{46}$ stands out. This solution represents a localized and exponentially decaying ultrasound wave $A$ which can scatter energy during the interaction with perturbations, in contrast to usual soliton solutions. The dromion arises when nonzero boundary conditions for the sound profile $Q$ are given and therefore exists due to energy exchange between sound and ultrasound waves.

Of even greater importance is the fact that the dromion (ultrasound wave) travels along the trajectory determined by the time dependence of the boundary conditions for the sound wave (at infinity). Setting the law that governs the variation of these boundary conditions, one can control the motion of the dromion.

Fokas and Santini ${ }^{46}$ showed that the DSI equations govern the evolution of $N$ dromions ( $N$ is natural) for arbitrary time-dependent boundary conditions for $Q$ and any initial 
conditions for $A$ decaying at infinity. To find a one-dromion solution it is necessary to rotate the system of coordinates through $45^{\circ}: x \rightarrow \tilde{x}+\tilde{y}, y \rightarrow \tilde{x}-\tilde{y}$. By introducing the variables $U \equiv\left(\partial Q / \partial x-|A|^{2}\right) / 2$ and $V \equiv\left(\partial Q / \partial y-|A|^{2}\right) / 2$ (the tilde is omitted), Eqs. (42) with $\nu=1$ can then be written in the form

$$
\begin{aligned}
& i \frac{\partial A}{\partial t}+\frac{\partial^{2} A}{\partial x^{2}}+\frac{\partial^{2} A}{\partial y^{2}}+(U+V) A=0, \quad \frac{\partial U}{\partial y}=\frac{\partial|A|^{2}}{\partial x}, \\
& \frac{\partial V}{\partial x}=\frac{\partial|A|^{2}}{\partial y} .
\end{aligned}
$$

Provided that the boundary conditions for $U$ on $y$ and $V$ on $x$ at $-\infty$ are of the form of one-dimensional $\mathrm{KdV}$ solitons

$$
\begin{aligned}
& \left.U\right|_{y \rightarrow-\infty}=\frac{8 k_{r}^{2} \exp \left(\eta_{1}+\eta_{1}^{*}\right)}{\left[1+\exp \left(\eta_{1}+\eta_{1}^{*}\right)\right]^{2}}, \\
& \left.V\right|_{x \rightarrow-\infty}=\frac{8 l_{r}^{2} \exp \left(\eta_{2}+\eta_{2}^{*}\right)}{\left[1+\exp \left(\eta_{2}+\eta_{2}^{*}\right)\right]^{2}},
\end{aligned}
$$

Eqs. (44) are easily integrated and give a one-dromion solution of the form ${ }^{47}$

$$
A=\frac{\chi \exp \left(\eta_{1}+\eta_{2}\right)}{1+\exp \left(\eta_{1}+\eta_{1}^{*}\right)+\exp \left(\eta_{2}+\eta_{2}^{*}\right)+\lambda \exp \left(\eta_{1}+\eta_{1}^{*}+\eta_{2}+\eta_{2}^{*}\right)} .
$$

Here

$$
\begin{aligned}
& \eta_{1}=\left(k_{r}+i k_{i}\right) x+\left(-2 k_{r} k_{i}+i \Omega_{i}\right) t, \\
& \eta_{2}=\left(l_{r}+i l_{i}\right) y+\left(-2 l_{r} l_{i}+i \omega_{i}\right) t, \\
& \Omega_{i}+\omega_{i}=k_{r}^{2}+k_{i}^{2}+l_{r}^{2}+l_{i}^{2}, \\
& \chi=2\left(2 k_{r} l_{r}(\lambda-1)\right)^{1 / 2},
\end{aligned}
$$

and $\lambda, k_{r}, k_{i}, l_{r}, l_{i}$ are arbitrary real numbers (parameters of the solution). The spatial distributions of the ultrasound envelope $|\widetilde{S}|=\gamma^{-1 / 2}|A|$ and of the sound profile $L=(U+V$ $\left.+2|A|^{2}\right) /(2 \delta)$, which correspond to the one-dromion solution given by Eqs. (45)-(47), are illustrated in Fig. 4.

The numerical experiments ${ }^{47}$ confirm the fact that the motion of the dromion structure occurs along the trajectory determined by the boundary conditions for the long wave. For example, when the variables in Eqs. (45) are changed as

$$
\begin{aligned}
& \eta_{1}+\eta_{1}^{*}=k_{r} x+\Omega_{r} \sin (w t), \\
& \eta_{2}+\eta_{2}^{*}=l_{r} x+\omega_{r} \cos (w t),
\end{aligned}
$$

i.e., the crosspoint of the sound wave (point $\mathrm{C}$ in Fig. 4) revolves around the origin, the dromion propagates quasistably around a circle.
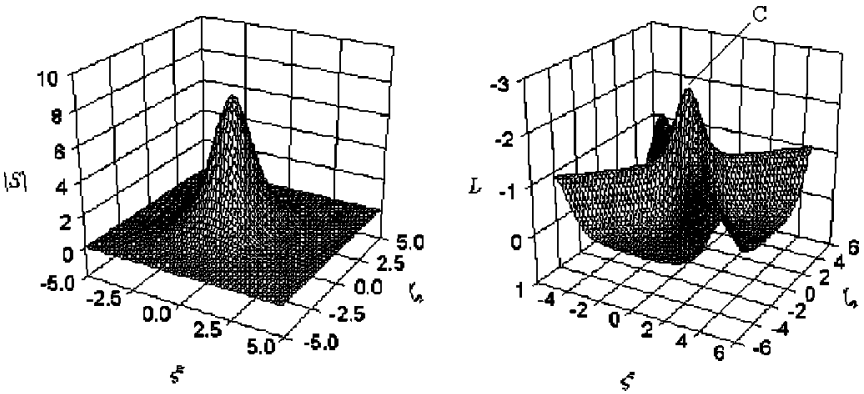

FIG. 4. One-dromion solution of the DSI equations with $\lambda=3, k_{r}=l_{r}$ $=4 / 5, k_{i}=l_{i}=1 / 5$.

\section{B. Singular focusing}

If the coefficients $\beta, \varrho, \sigma, \alpha \delta$ are positive and $\gamma>-\alpha \delta / c_{e}^{2}$, the solution to Eqs. (41) with the boundary condition

$$
|\widetilde{S}| \rightarrow 0 \quad \text { for } \xi^{2}+\zeta^{2} \rightarrow \infty,
$$

tends to infinity for reasonably large amplitudes within a finite time interval. ${ }^{25}$ This phenomenon is often referred to as singular focusing or blowup instability of two-dimensional wave perturbations. There are experimental data ${ }^{48}$ which confirm the existence of the singular focusing in nonlinear optics.

The singular focusing of the solutions to Eqs. (41) is corroborated by the analysis of conservation laws. From Eqs. (41) it follows that the integrals:

$$
\begin{aligned}
M= & \iint_{-\infty}^{+\infty}|\widetilde{S}|^{2} d \xi d \zeta, \\
P_{x}= & \iint_{-\infty}^{+\infty}\left(\widetilde{S^{*}} \frac{\partial \widetilde{S}}{\partial \xi}-\widetilde{S} \frac{\partial \widetilde{S}^{*}}{\partial \xi}\right) d \xi d \zeta, \\
P_{y}= & \iint_{-\infty}^{+\infty}\left(\widetilde{S^{*}} \frac{\partial \widetilde{S}}{\partial \zeta}-\widetilde{S} \frac{\partial \widetilde{S}^{*}}{\partial \zeta}\right) d \xi d \zeta, \\
E= & \iint_{-\infty}^{+\infty}\left[\beta\left|\frac{\partial \widetilde{S}}{\partial \xi}\right|^{2}+\varrho\left|\frac{\partial \widetilde{S}}{\partial \zeta}\right|^{2}\right. \\
& \left.-\frac{1}{2}\left(\gamma|\widetilde{S}|^{4}+\frac{\sigma c_{e}^{2}}{\alpha \delta}\left|\frac{\partial \Psi}{\partial \xi}\right|^{2}+\frac{c_{e}^{2}}{\alpha \delta}\left|\frac{\partial \Psi}{\partial \zeta}\right|^{2}\right)\right] d \xi d \zeta,
\end{aligned}
$$

are conserved. The first integral is the "mass" of the short wave. The next two integrals are the $\xi$-and $\zeta$-components of the short-wave "momentum." We obtain (49) and (50) by multiplying the second equation of (41), respectively into $2 \widetilde{S}^{*}, \widetilde{S}_{\xi}^{*}$, and $\widetilde{S}_{\zeta}^{*}$, then taking the real parts of the results, and integrating them with respect to $\xi$ and $\zeta$ between $-\infty$ 


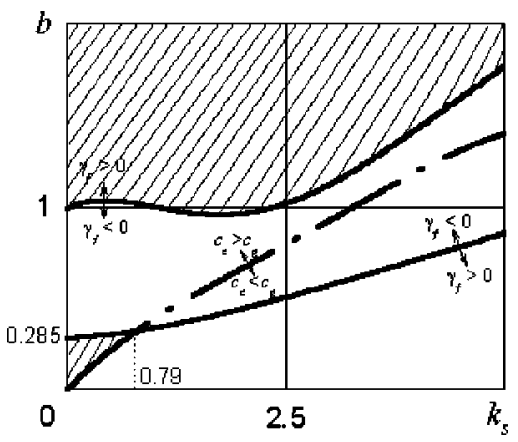

FIG. 5. Regions of the blowup instability $\left(\gamma_{f}=\gamma+\alpha \delta / c_{e}^{2}\right)$.

and $\infty$ using the boundary condition (48). The integral (51) may be interpreted as the "total energy" of interacting long and short waves. The "energy" can be negative under the condition of singular focusing and large initial amplitudes of ultrasound waves. The steps of deriving (51) are following: (i) The second equation of (41) is multiplied into $2 \widetilde{S}_{\tau}^{*}$ and the real part of the result is considered; (ii) the real part contains the interaction term

$$
\frac{\partial|\widetilde{S}|^{2}}{\partial \tau} \frac{\partial \Psi}{\partial \xi}
$$

which is calculated using the first equation of (41); (iii) the obtained expression is integrated with the use of (48).

From (41) it follows also the relation:

$$
\frac{d^{2} I}{d t^{2}}=8 E, \quad I=\iint_{-\infty}^{+\infty}\left(\frac{\xi^{2}}{\varrho}+\frac{\zeta^{2}}{\beta}\right)|\widetilde{S}|^{2} d \xi d \zeta
$$

where $I$ is the "inertia moment" (a positive-definite magnitude). The derivation of (52) is not trivial and is shown in detail by Ghidaglia and $\mathrm{Saut}^{24}$ (see also Ref. 25). When $E$ $<0$, the "virial theorem" (52) causes the "inertia moment" to vanish in a finite time. Physically, this means that the
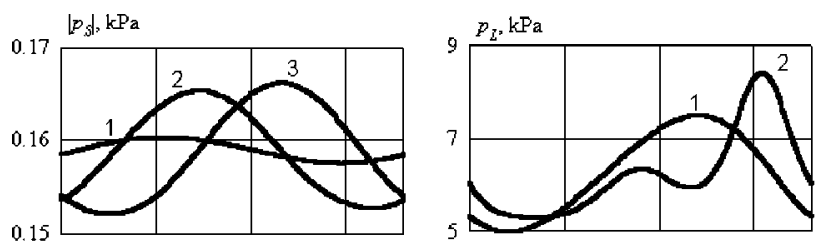

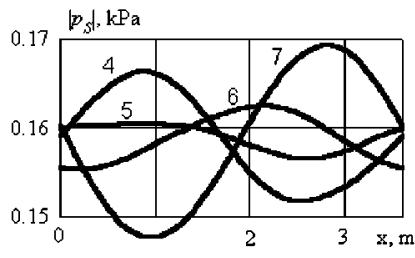

(a)

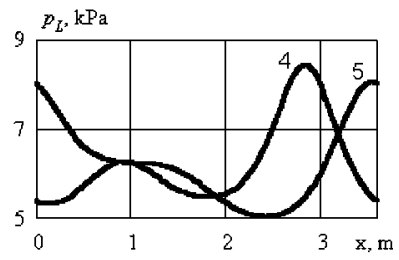

(b)
FIG. 6. Nondissipative evolution of (a) the sound wave, $p_{L}=\varepsilon^{l} p_{0} L$, and (b) the ultrasound wave packet, $\left|p_{S}\right|=\varepsilon^{s} p_{0}|S|$, according to Eqs. (24). Water with air bubbles of the radius $1 \mathrm{~mm}$ under normal conditions and $\varepsilon=0.1$, $L_{0}=S_{0}=5, \Delta L=0.25, \Delta S=0.05, \alpha_{g 0}=3 \times 10^{-4}, \mu=0$. The curves labeled $1,2,3,4,5,6$, and 7 are for the instants of time $t=0.019,0.171,0.209$, $0.294,0.437,0.532$, and $0.722 \mathrm{~s}$, respectively. Here $t=\tau / \varepsilon^{2}$ and $x$ $=\left(a_{0} \alpha_{g 0}^{-1 / 2} / \varepsilon\right) \xi$.

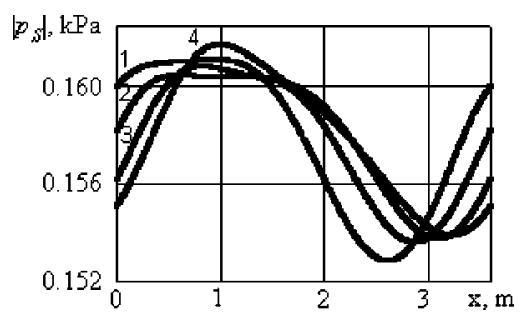

FIG. 7. Nonlinear distortions of the ultrasound wave packet. The values of parameters are listed in Fig. 6. The curves labeled 1, 2, 3, and 4 are for the instants of time $t=0.4557,0.4652,0.4747$, and $0.4842 \mathrm{~s}$, respectively.

ultrasonic perturbation distributed initially in space will be concentrated at some spatial point with time. Since the mass of the ultrasound wave is conserved, this process leads to increasing the wave amplitude at the spatial point and, finally, results in the blowup instability.

In the bubbly fluid, the coefficients $\beta, \varrho$ are always positive, and $\alpha$ and $\delta$ are of the same sign. Hence, the singular focusing of ultrasound is possible in the bubbly mixtures if ${ }^{49}$

$$
c_{e}>c_{g}, \quad \gamma>-\alpha \delta / c_{e}^{2} .
$$

The regions of the blowup instability, determined by condition (53), are plotted in Fig. 5.

\section{NUMERICAL ANALYSIS}

\section{A. Degenerate resonant interaction}

The models (28) and (32) are integrated numerically using a three-layer explicit scheme with a fourth order of approximation with respect to the spatial coordinate proposed earlier ${ }^{50}$ for the numerical solution of the KdV equation (see details in the Appendix). Periodic boundary conditions and an initial condition of the form

$$
\begin{aligned}
& \left.L\right|_{\tau=0}=L_{0}(1+\Delta L[1-\cos \xi]), \\
& \left.S_{r}\right|_{\tau=0}=S_{0}(1+\Delta S[1-\cos \xi]),\left.\quad S_{i}\right|_{\tau=0}=0,
\end{aligned}
$$

where $S_{r}=\operatorname{Re}(S) ; S_{i}=\operatorname{Im}(S)$, are used in the integration.

The results of numerical investigation of (28) are represented in Figs. 6-8. Figure 6 shows the spatial distributions of the ultrasound envelope and the sound profile (damping is not considered). It is clear that a sinusoidal profile for $|S|$ is

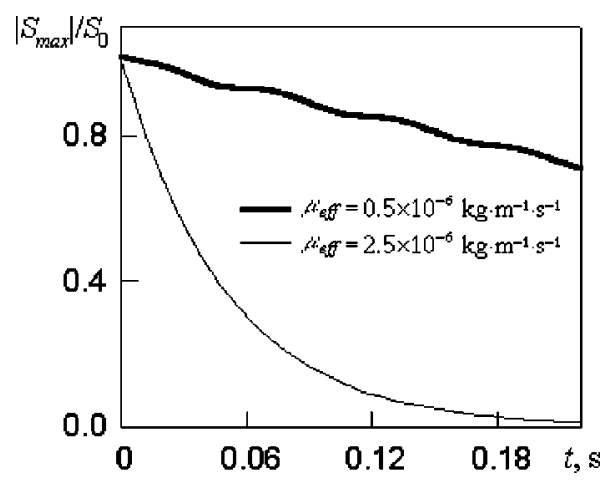

FIG. 8. The maximal value of the ultrasound amplitude versus time. The other parameters are the same as in Fig. 6. 


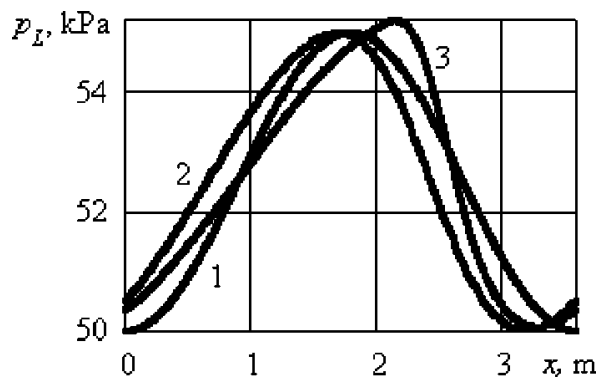

FIG. 9. Tendency to a shock wave formation in model (26). Here $\varepsilon=0.1$, $L_{0}=S_{0}=5, \Delta L=\Delta S=0.05, p_{0}=0.1 \mathrm{MPa}, \kappa=1.4$. The curves labeled 1, 2, and 3 are for the instants of time $t=0,0.133$, and $0.285 \mathrm{~s}$.

formed. The amplitude of this profile increases up to the instant of time $t=0.294 \mathrm{~s}$ and then sharply decreases at $t$ $=0.437 \mathrm{~s}$, and, after a certain time, it increases again, reaching a maximum value at $t=0.722 \mathrm{~s}$ [Fig. 6(a)]. In the case of sinusoidal initial conditions ultrasound has no action on sound and the sound wave propagates in accordance with the $\mathrm{KdV}$ equation (19). It is important to note that an increase in the ultrasound amplitude occurs during the formation of a second hump in the sound profile (the two-soliton solution of the KdV equation) and a decrease happens when the hump disappears [Fig. 6(b)]. From this it follows that the formation
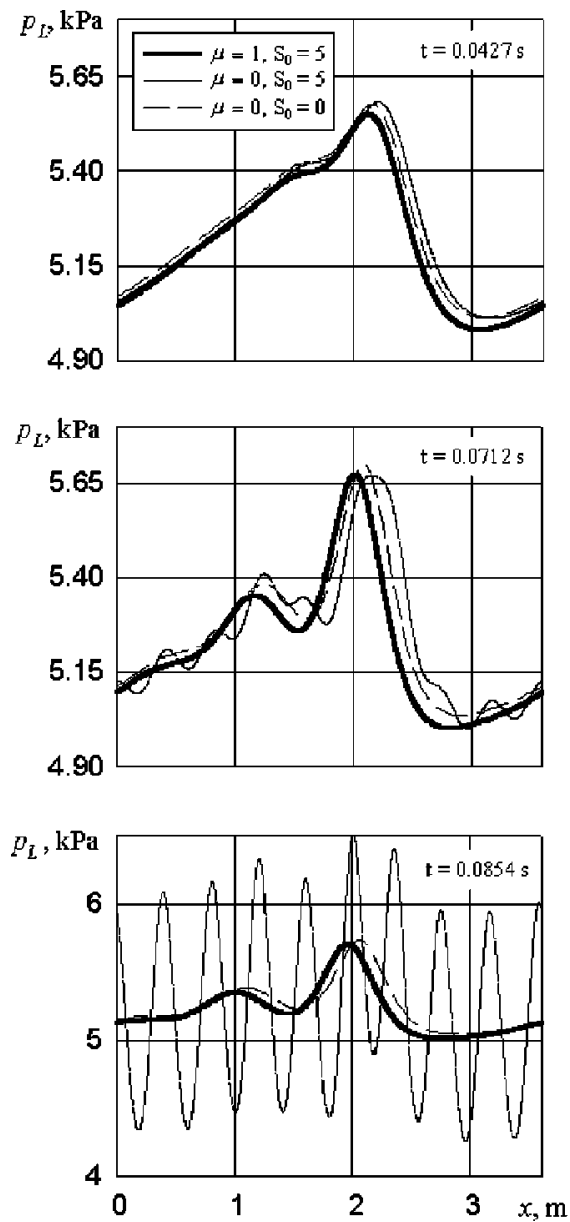

FIG. 10. Evolution of the sound perturbation $p_{L}=\varepsilon^{l} p_{0} L$. The parameters are given in the legend and in Fig. 9.

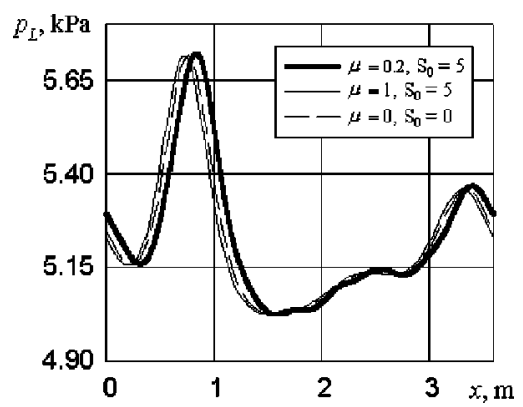

FIG. 11. Spatial distribution of the sound perturbation according to Eqs. (26) for different values of the damping coefficient.

of a two-humped profile is accompanied by a significant return flow of energy from sound to ultrasound, on account of which, in spite of strong dispersion, the amplitude of the ultrasound wave increases. The return flow of energy is provided by the interaction terms $L \partial S / \partial \xi$ and $S \partial L / \partial \xi$. When $L$ increases or, at least, does not decrease at some spatial point $\xi=\xi_{*}$, these terms ensure a positiveness of the derivative $\partial S / \partial \tau$ and, hence, increasing the ultrasound amplitude at the point $\xi_{*}$ in time. Going through the periods of growth and fall, this amplitude grows on the whole which is seen from a comparison of the ultrasonic distribution at the instants of time $t=0.209 \mathrm{~s}$ and $t=0.722 \mathrm{~s}$ [curves 3 and 7 in Fig. 6(a)]. The essential smallness and briefness of the nonlinear distortions in the ultrasound profile should be noted. These distortions appear when the amplitude of ultrasound reduces [cf. Fig. 7]. Consequently, we have the linear intensification of ultrasound by sound here.

The results obtained can be given the following physical interpretation. Suppose that quasimonochromatic highfrequency (ultrasonic) and low-frequency (sonic) oscillations are excited in a bubbly fluid. In general, these perturbations will propagate in the medium in the form of an ultrasound wave packet and a sound wave. When the long-wave-shortwave resonance condition is satisfied, interaction occurs which obeys the Zakharov equations (24). This system describes the formation of a soliton structure for the given boundary-initial conditions (in the nondissipative case). ${ }^{45}$ Obviously, the soliton is a consequence of significant nonlinear distortions in the ultrasound profile. However, an isolated ultrasound frequency exists in a bubbly fluid, that is, the frequency of "degeneracy" at which the resonant interaction has a completely different character. First, the sound wave propagates independently of the ultrasound wave and obeys the KdV equation. Second, due to the action of the sound wave on the ultrasound wave a sinusoidal profile of the ultrasound envelope is formed. This profile experiences negligible and short-term nonlinear distortions. This means that practically all the energy of ultrasound will be contained in its first harmonic. Third, due to the flow of energy from the sound wave to the ultrasound wave, the growth of the shortwave amplitude takes place.

What happens if we add damping? Restrict our attention to very small damping $\left(\mu^{*}=\varepsilon^{3} \mu\right)$ because small one ( $\mu^{*}$ $=\varepsilon^{2} \mu$ ) suppresses any interaction in this model (cf. above). Of course, the very weak damping leads to the attenuation of 
$t=0$
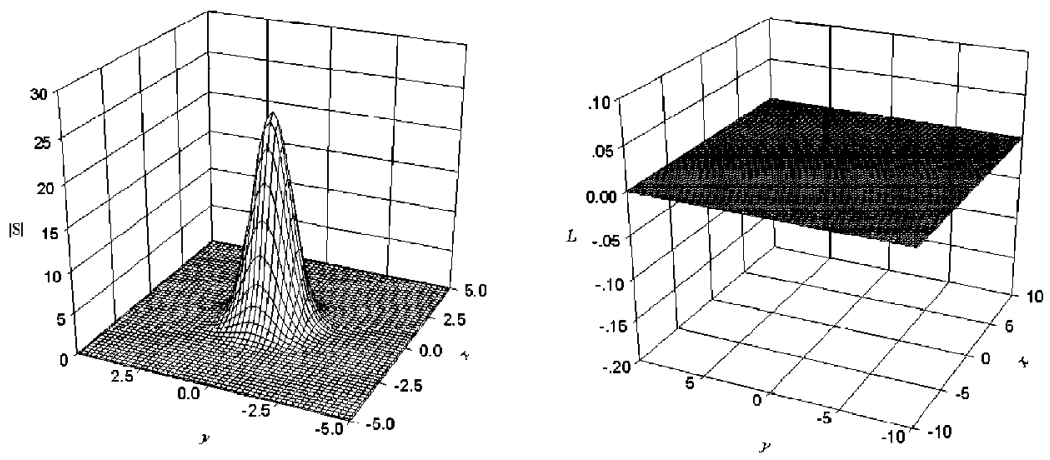

$t=0.39633$
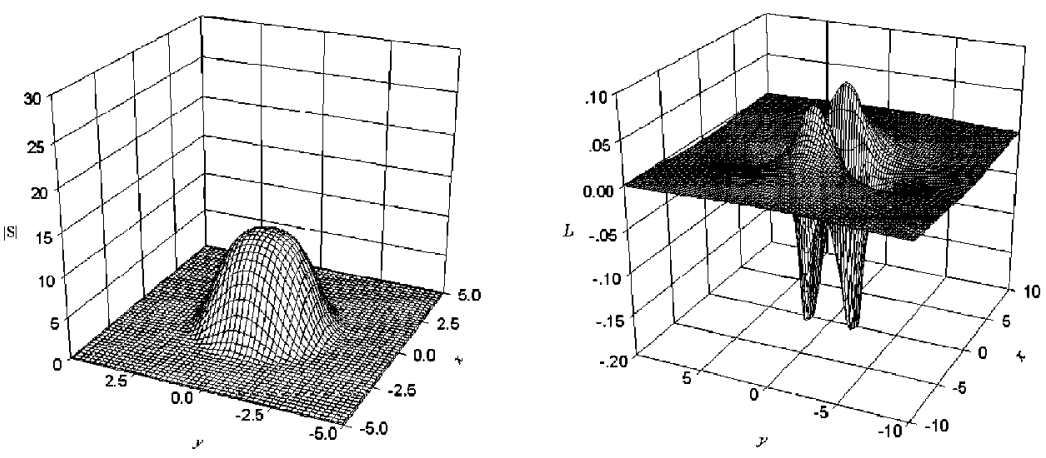

FIG. 12. Nonresonant defocusing of ultrasound and sound in a bubbly fluid $\left(S_{0}=25, \kappa=1.4\right)$.

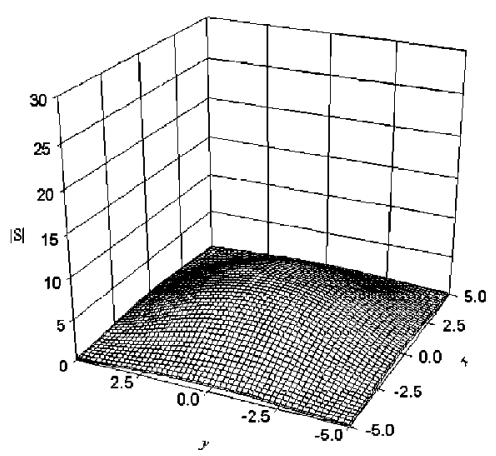

$t=0.9936$

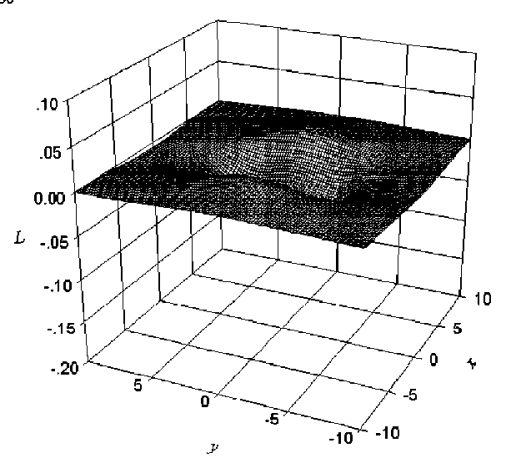

ultrasonic perturbations. If $\mu$ is large enough, the amplitude of the short wave decreases exponentially in time (thin solid line in Fig. 8). The interaction between sound and ultrasound waves appears for a smaller damping coefficient. Due to the interaction, the attenuation curve of the ultrasound amplitude becomes of graduated type (thick solid line in Fig. 8). A slower attenuation is due to increasing the ultrasound amplitude (in the nondissipative case).

The absolutely different situation is observed for model (32). Figure 9 shows the sonic distribution with respect to $x$ at $\mu=0$ at different instants of time. It is clear that sound propagates with its profile becoming steeper since, when no account is taken of terms of order $\varepsilon$, the equation for the sound wave is the Hopf equation (34).

Figures 10 and 11 represent the subsequent evolution of the sonic and ultrasonic perturbations in the presence (thick line) and absence (thin and dashed lines) of weak damping. A shock profile is not formed. This is due to the contribution of terms of order $\varepsilon$ in the first equation of (32). If the ultrasonic perturbations are not generated $\left(S_{0}=0\right)$, the sound wave will propagate in accordance with the strongly nonlinear $\mathrm{KdV}$ equation

$$
\frac{\partial L}{\partial \tau}+\sigma \frac{\partial L^{2}}{\partial \xi}+\varepsilon \varsigma \frac{\partial L^{3}}{\partial \xi}+\varepsilon \chi \frac{\partial^{3} L}{\partial \xi^{3}}=0
$$

(Fig. 10, dashed line). But if $S_{0}>0$, the sound profile fails (Fig. 10, thin line). Thus, the nondissipative interaction between high intensity sound and ultrasound results in nonlinear instability in the case of resonant degeneracy. Note that this instability appears due to the tendency to a shock wave formation and the presence of the terms with cubic nonlinearity, $\varrho \partial\left(L|S|^{2}\right) / \partial \xi$ and $\widetilde{\delta} L^{2} S$, in Eqs. (32). The values of the sound profile are quite different in two close points on the shock. This difference is doubled and transmitted to the ultrasound wave due to the term $\widetilde{\delta} L^{2} S$ and leads to the further steepness of the ultrasound profile. Due to the term $\varrho \partial\left(L|S|^{2}\right) / \partial \xi$ the originating jump in $S$ influences the sound wave and, finally, results in the nonlinear instability. 


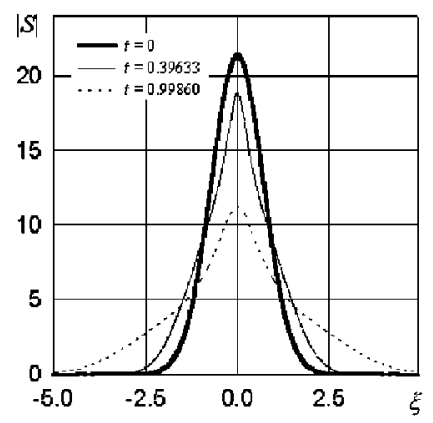

(a)

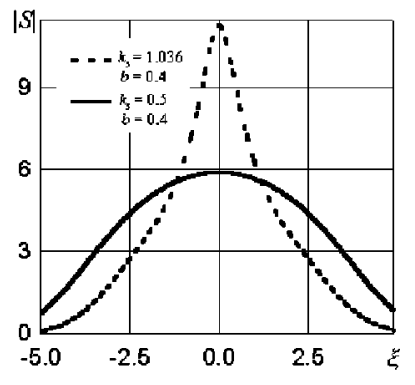

(b)
FIG. 13. Defocusing of ultrasound in the resonant case: (a) the ultrasound envelope $|S|$ versus $\xi(\zeta=0)$ at different instants of time; (b) comparison of the nonresonant (thick curve) and resonant (dashed curve) ultrasound profiles at $\tau=1$.

Weak damping suppresses the nonlinear instability and results in insignificant decreasing the amplitude and velocity of a solitary sound wave. The disappearance of the instability comes from the fast attenuation of the ultrasonic signal: The contribution of $S$ into $L$ becomes small even during the steepness of the ultrasonic distribution. Nevertheless, if we reduce the damping coefficient, the nonlinear instability will arise again (Fig. 11, thick curve). This is because the ultrasound amplitude decreases slowly now, and nonlinear distortions are large enough for the destruction of the sound profile.

\section{B. Singular focusing}

The results of numerical analysis of Eqs. (35) are illustrated in Figs. 12-15. The first equation is solved by the Fourier transform method, ${ }^{51}$ the second equation by the alternating-direction implicit method. ${ }^{52}$ The boundary and initial conditions are as follows:

$$
L(\xi, \zeta), \quad S(\tau, \xi, \zeta)=0, \quad \text { for } \xi^{2}+\zeta^{2} \rightarrow \infty
$$

and

$$
S(\tau=0, \xi, \zeta)=S_{0} \exp \left\{-\left(\xi^{2}+\zeta^{2}\right)\right\} .
$$

Here $S_{0}$ takes real values. The coefficients in (35) are selected so that $c_{e}>c_{g}$ [then the first equation of (35) is of elliptic type]. We analyze four cases: (1) $k_{s}=0.5, b=0.4$; (2) $k_{s}=1.036, b=0.4$; (3) $k_{s}=0.5, b=0.25$; (4) $k_{s}=0.574, b$ $=0.25$. The first two cases are considered on the assumption that $\mu=0$. The first case corresponds to the stable nonresonant interaction because $c_{e} \neq c_{g}$ and point $(0.5 ; 0.4)$ lies in the stable region (cf. Fig. 5).

Numerical analysis confirms analytical results. The stable interaction leads to defocusing of sound and ultrasound waves (Fig. 12). In the second case we are also in the stable region but near the resonant curve $\left(c_{g} \rightarrow c_{e}\right)$. The numerical solution of (35) defocuses again [Fig. 13(a)]. But now the defocusing slows down and is accompanied by the modification of the wave profile [Fig. 13(b)].

In the third case the unstable nonresonant interaction takes place. Indeed, with no damping the high intensity ultrasound wave $\left(S_{0}>40\right)$ is shrinking and growing in time (Fig. 14). This results in the blowup instability later. Weak damping suppresses this instability until $\mu$ exceeds some cutoff value. It should be noted that a similar cut-off influence of damping on singular focusing was observed for the generalized $\mathrm{KdV}$ equation with nonlinearities of fourth order. $^{53}$

The singular focusing of nondissipative interaction is evident in the fourth (unstable resonant) case. Now the focusing is accelerated and accompanied by the strong deformation of the ultrasound profile (Fig. 15). Also, the amplitude threshold for singular focusing reduces to $S_{0}=20$. Hence the long-wave-short-wave resonance enhances the probability of singular focusing of ultrasound in bubbly fluids.

\section{POSSIBLE APPLICATIONS}

In conclusion, we would like to outline possible applications of such sound-ultrasound interactions.

First, the phenomenon of degeneracy of the interaction can be used for ultrasound diagnostics of weakly viscous bubbly fluids in narrow channels. Narrow channels are essential here in order that transverse instabilities do not evolve. If we find some value of the degeneracy frequency experimentally, we can evaluate the radius of the bubble, $a_{0 d}$, and the volume gas content $\alpha_{g 0 d}$. Indeed, from condition (29) and formulas (2) and (4a) it follows that $\alpha_{g 0 d}$ depends only on the ambient pressure $p_{0}$, mass density of a carrier liquid, $\rho_{l 0}$, speed of sound in the liquid, $C_{l}$, and polytropic exponent $\kappa$ :

$$
\alpha_{g 0 d}=\frac{p_{0} \kappa^{2}(3 \kappa+2)^{2}}{\rho_{l 0} C_{l}^{2}[3 \kappa(\kappa+1)+1]} .
$$

If $\alpha_{g 0 d}$ is known, there is no problem to find $a_{0 d}$ because

$$
a_{0 d}=\frac{3}{2 \pi f_{s d}} \sqrt{\frac{p_{0} \kappa(\kappa+1)}{\rho_{l 0}\left(1-\alpha_{g 0 d}\right)}},
$$

where $f_{s d}$ is the degeneracy frequency. Of course, actual bubbly fluids are polydispersed. This requires to include the bubble size distribution in the equations of motion. There are two changes in the equations for perturbations (3) if the bubbly fluid becomes polydisperse (see Ref. 16). First, we have

$$
\sum_{j=2}^{N} \frac{\alpha_{j 0}}{\alpha_{g 0}}\left(1+a_{j}\right)^{3},
$$

instead of $(1+a)^{3}$ in Eq. (3b). Here $N-1$ is the number of disperse (or bubble) fractions $(N=1$ corresponds to the dispersive medium), $\alpha_{j 0}$ and $a_{j}$ are the initial volume content and perturbation in the bubble radius for $j$ th fraction, $\alpha_{g 0}$ $=\sum_{j=2}^{N} \alpha_{j 0}$ is the total initial volume gas content. Second, in Eq. (3c), $a$ is replaced by $a_{j}$ and the first two terms are multiplied by

$$
\xi_{j}=\frac{a_{j 0}}{a_{*}}
$$

( $a_{j 0}$ is the initial bubble radius for $j$ th fraction, $a_{*}$ is a certain representative radius of bubbles). There are $N+1$ equations for perturbations at all: The first is for the pressure perturbation, the second is for the density perturbation, and 


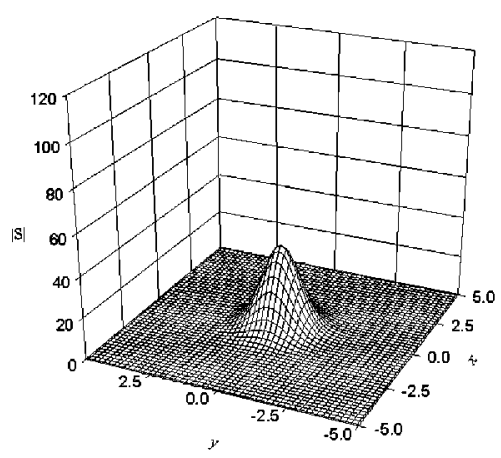

$t=0$

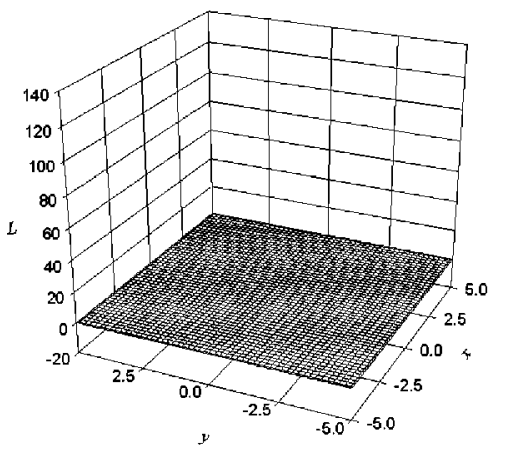

$t=0.09714$
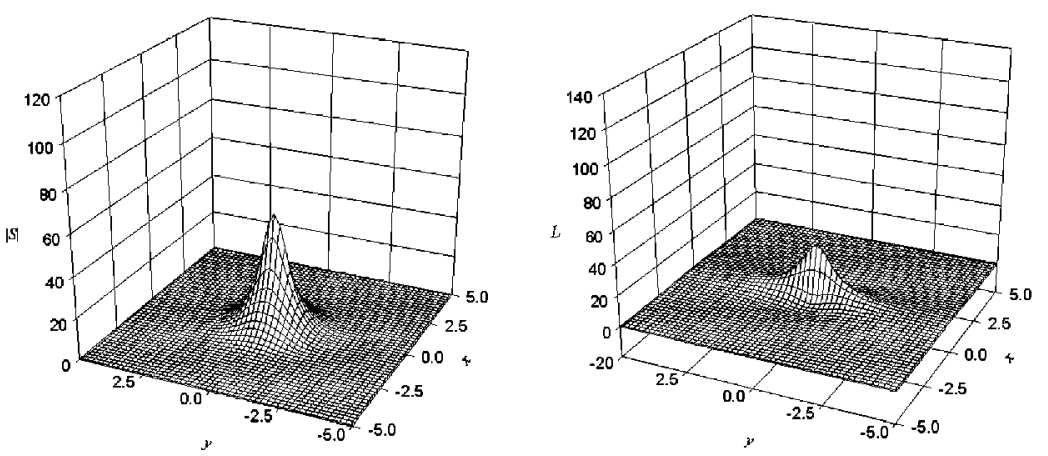

$t=0.15931$
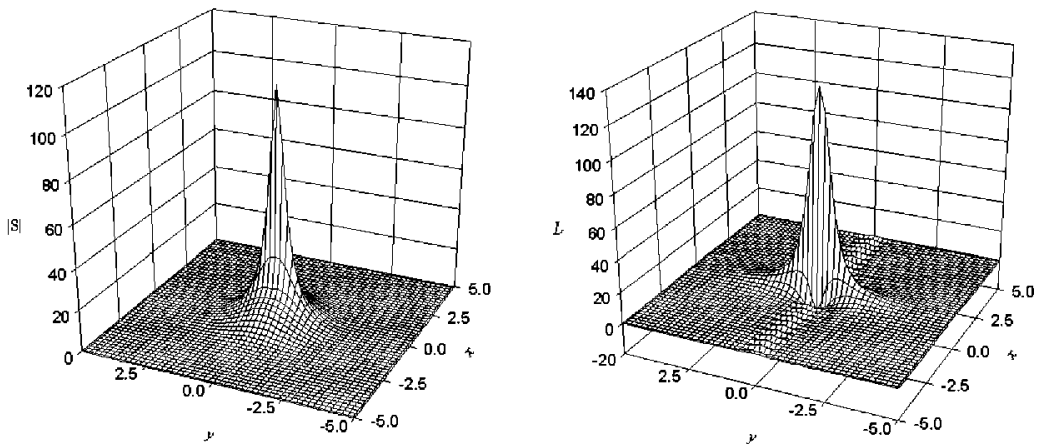

FIG. 14. Nonresonant singular focusing of ultrasound and sound in a bubbly fluid $\left(S_{0}=30 ; \kappa=1.4 ; \mu=0\right)$. the remaining $N-1$ equations are for the perturbations in bubble radii. Anyway, the equations have the same form, and hence the above results should be valid for polydisperse bubbly fluids. For example, there exists the long-wave-short-

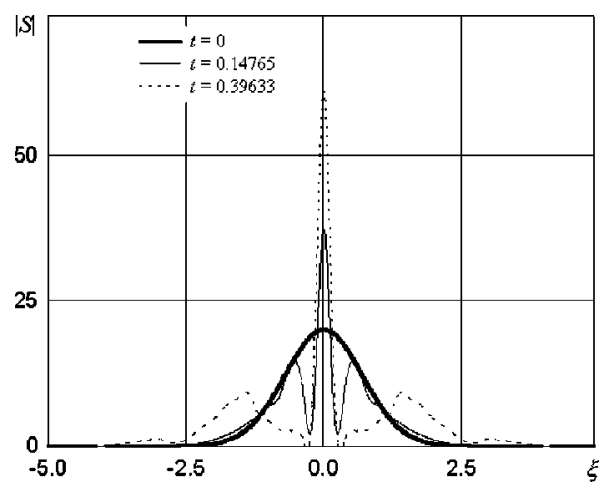

FIG. 15. Spatial distribution of the ultrasound envelope $|S|$ at different instants of time in the case of resonant singular focusing. Here $S_{0}=20 ; \kappa$ $=1.4, \mu=0$. wave resonance and the nonresonant one-dimensional dynamics is governed by the nonlinear Schrödinger equation. ${ }^{16}$

The main problem is to "catch" the degeneracy frequency. To do this the following ideas can be exploited. Usually, if we create ultrasound and sound in bubbly fluids simultaneously, so that resonance condition (10) is satisfied, these waves will interact according to the Zakharov equations with damping (24). The result of this interaction is the formation and the subsequent decay of the ultrasound envelope and the appearance of an additional sonic (long-wave) component proportional to a square of the ultrasound envelope modulus. Since weak damping leads to decreasing the ultrasound amplitude, the additional long-wave component will be also damped out but not so fast as in the degenerate case. Under degeneracy condition (29), ultrasound will not influence the long wave essentially. This gives us a chance to verify the degeneracy phenomenon experimentally. The experimental setup can be designed on a basis of the difference-frequency sound generation technique. ${ }^{12,54}$

Second, the dromion property to travel on the tracks, 
defined by the evolution of sound wave, can be drawn on to control ultrasonic action processes in bubbly fluids. If the ultrasonic disturbance is created in a liquid with gas bubbles, the parameters of which satisfy the existence of the dromion, there is then a possibility to focus the disturbance at some point of space and "remotely" control its motion by sound (on significant distance from the focus point).

Third, singular focusing of ultrasound is possibly a very dangerous phenomenon, especially in the case of longwave-short-wave resonance. It may result in explosions, violent cavitation in a liquid with microbubbles, for example, in human tissue, blood, and ocean water. Currently, the wide range of potential medical applications of high intensity focused ultrasound (HIFU), such as lithotripsy, ${ }^{55}$ cancer therapy, ${ }^{56}$ and gene therapy, ${ }^{57}$ is being developed. It should be noted that HIFU must be exploited in tissue in circumstances where the blowup instability condition is not satisfied.

The above-listed applications of the results invite further theoretical and experimental investigations. Our following efforts will be aimed at the elucidation of more complex theoretical models and the performance of required experiments.

\section{ACKNOWLEDGMENTS}

This material is based upon work supported by the European Commission under INCO-Copernicus program No. ERBIC15CT980141 and the North Atlantic Treaty Organization under Grant No. 0000779. Any opinions, findings, conclusions or recommendations expressed in this publication are those of the authors and do not necessarily reflect the view of NATO.

\section{APPENDIX: NUMERICAL METHOD FOR SOLVING DEGENERATE EQUATIONS}

The equations for resonant degenerate interaction (28) and (32) have been solved numerically using a three-layer explicit scheme with a fourth order of approximation with respect to the spatial coordinate. ${ }^{50}$ In the appendix, the finitedifference approximation of only Eqs. (28) is presented. The discrete equations of the second model are too cumbersome to be given here. According to the scheme, the equation for the long-wave profile $L$ looks as follows:

$$
\begin{aligned}
L_{j}^{n+1}= & L_{j}^{n-1}+\frac{\sigma \Delta t}{3 \Delta x} L_{j}^{n}\left[L_{j+2}^{n}-8 L_{j+1}^{n}+8 L_{j-1}^{n}-L_{j-2}^{n}\right] \\
& -\frac{\lambda \Delta t}{3(\Delta x)^{2}} S i_{j}^{n}\left[S r_{j+2}^{n}-16 S r_{j+1}^{n}+30 S r_{j}^{n}\right. \\
& \left.-16 S r_{j-1}^{n}+S r_{j-2}^{n}\right]+\frac{\lambda \Delta t}{3(\Delta x)^{2}} S r_{j}^{n}\left[S i_{j+2}^{n}\right. \\
& \left.-16 S i_{j+1}^{n}+30 S i_{j}^{n}-16 S i_{j-1}^{n}+S i_{j-2}^{n}\right] \\
& +\frac{\chi \Delta t}{4(\Delta x)^{3}}\left[L_{j+3}^{n}-8 L_{j+2}^{n}+13 L_{j+1}^{n}-13 L_{j-1}^{n}\right.
\end{aligned}
$$

$$
\left.+8 L_{j-2}^{n}-L_{j-3}^{n}\right] \text {. }
$$

Because the short-wave envelope is complex-valued ( $S$ $=S r+i S i$ ), Eq. (28b) breaks down into the following discrete equations:

$$
\begin{aligned}
S r_{j}^{n+1}= & S r_{j}^{n-1}-\frac{\mu \Delta t}{6 \Delta x} L_{j}^{n}\left[S r_{j+2}^{n}-8 S r_{j+1}^{n}+8 S r_{j-1}^{n}\right. \\
& \left.-S r_{j-2}^{n}\right]-\frac{\nu \Delta t}{6 \Delta x} S r_{j}^{n}\left[L_{j+2}^{n}-8 L_{j+1}^{n}+8 L_{j-1}^{n}\right. \\
& \left.-L_{j-2}^{n}\right]+2 \gamma \Delta t S i_{j}^{n}\left[\left(S r_{j}^{n}\right)^{2}+\left(S i_{j}^{n}\right)^{2}\right] \\
& +\frac{\widetilde{\beta} \Delta t}{6(\Delta x)^{2}}\left[S i_{j+2}^{n}-16 S i_{j+1}^{n}+30 S i_{j}^{n}-16 S i_{j-1}^{n}\right. \\
& \left.+S i_{j-2}^{n}\right]+\frac{\eta \Delta t}{4(\Delta x)^{3}}\left[S r_{j+3}^{n}-8 S r_{j+2}^{n}+13 S r_{j+1}^{n}\right. \\
& \left.-13 S r_{j-1}^{n}+8 S r_{j-2}^{n}-S r_{j-3}^{n}\right]
\end{aligned}
$$

and

$$
\begin{aligned}
S i_{j}^{n+1}= & S i_{j}^{n-1}-\frac{\mu \Delta t}{6 \Delta x} L_{j}^{n}\left[S i_{j+2}^{n}-8 S i_{j+1}^{n}+8 S i_{j-1}^{n}\right. \\
& \left.-S i_{j-2}^{n}\right]-\frac{\nu \Delta t}{6 \Delta x} S i_{j}^{n}\left[L_{j+2}^{n}-8 L_{j+1}^{n}+8 L_{j-1}^{n}\right. \\
& \left.-L_{j-2}^{n}\right]-2 \gamma \Delta t S r_{j}^{n}\left[\left(S r_{j}^{n}\right)^{2}+\left(S i_{j}^{n}\right)^{2}\right] \\
& -\frac{\widetilde{\beta} \Delta t}{6(\Delta x)^{2}}\left[S r_{j+2}^{n}-16 S r_{j+1}^{n}+30 S r_{j}^{n}-16 S r_{j-1}^{n}\right. \\
& \left.+S r_{j-2}^{n}\right]+\frac{\eta \Delta t}{4(\Delta x)^{3}}\left[S i_{j+3}^{n}-8 S i_{j+2}^{n}+13 S i_{j+1}^{n}\right. \\
& \left.-13 S i_{j-1}^{n}+8 S i_{j-2}^{n}-S i_{j-3}^{n}\right] .
\end{aligned}
$$

Here subscripts and superscripts denote spatial and temporal locations, $\Delta t$ and $\Delta x$ are the time and spatial steps, $t=t_{3}$ $=\varepsilon \tau, x=\xi, L_{j}^{n}=L\left(x_{j}, t_{n}\right), S r_{j}^{n}=S r\left(x_{j}, t_{n}\right), S i_{j}^{n}=S i\left(x_{j}, t_{n}\right)$, $x_{j}=(j-1) \Delta x, t_{n}=n \Delta t$, and $\widetilde{\beta}=\beta / \varepsilon$. The indexes $j$ and $n$ take the values from 1 and 0 to $J$ and $N$, respectively.

The boundary conditions are periodic

$$
\begin{array}{ll}
L_{0}^{n+1}=L_{J}^{n+1}, & L_{J+1}^{n+1}=L_{1}^{n+1}, \quad L_{J+2}^{n+1}=L_{2}^{n+1}, \\
L_{J+3}^{n+1}=L_{3}^{n+1}, & \\
S r_{0}^{n+1}=S r_{J}^{n+1}, & S r_{J+1}^{n+1}=S r_{1}^{n+1}, \\
S r_{J+2}^{n+1}=S r_{2}^{n+1}, & S r_{J+3}^{n+1}=S r_{3}^{n+1}, \\
S i_{0}^{n+1}=S i_{J}^{n+1}, & S i_{J+1}^{n+1}=S i_{1}^{n+1}, \\
S i_{J+2}^{n+1}=S i_{2}^{n+1}, & S i_{J+3}^{n+1}=S i_{3}^{n+1} .
\end{array}
$$

The stability analysis for the numerical scheme has been performed using the Neumann method. ${ }^{52}$ According to this method, we assume that

$$
\begin{aligned}
& L_{j}^{n-1}=L^{\prime} e^{a t+i k x}, \quad S r_{j}^{n-1}=S r^{\prime} e^{a t+i k x}, \\
& S i_{j}^{n-1}=S i^{\prime} e^{a t+i k x},
\end{aligned}
$$




$$
\begin{aligned}
& L_{j}^{n}=L_{j}^{n-1} e^{a \Delta t}, \quad S r_{j}^{n}=S r_{j}^{n-1} e^{a \Delta t}, \quad S i_{j}^{n}=S i_{j}^{n-1} e^{a \Delta t}, \\
& L_{j+1}^{n-1}=L_{j}^{n-1} e^{i k \Delta x}, \quad S r_{j+1}^{n-1}=S r_{j}^{n-1} e^{i k \Delta x}, \\
& S i_{j+1}^{n-1}=S i_{j}^{n-1} e^{i k \Delta x} .
\end{aligned}
$$

Substitution of Eqs. (A4) into the finite-difference equations leads to a system of algebraic equations in $L_{j}^{n-1}, S r_{j}^{n-1}$, and $S i_{j}^{n-1}$ for each of the models. From the compatibility condition we obtain a polynomial equation in $\exp (a \Delta t)$. The stability criterion follows from solving this equation and is based on the fact that the linear instability evolves when $\exp (a \Delta t)>1$.

The stability analysis for Eqs. (A1)-(A3) results in the polynomial equation of the fourth order which has rather unwieldy solutions. We have examined the approximate solutions to this equation using Mathematica and obtained the following stability criterion at $\widetilde{\beta} \approx 4.9$ and $\eta \approx 0.06$ :

$$
\Delta t \leqslant \min \left(0.042 \Delta x^{3}, \frac{0.216 \Delta x^{3}}{\chi}\right) .
$$

The stability criterion for the finite-difference equations of (32) is even simpler

$$
\Delta t \leqslant \min \left(\frac{3 \sqrt{2} \Delta x^{2}}{32 \beta}, \frac{0.216 \Delta x^{3}}{\varepsilon \chi}\right) .
$$

${ }^{1}$ G. A. Askar'yan, "Self-focusing of powerful sound during the production of bubbles," JETP Lett. 13, 283 (1971).

${ }^{2}$ P. Giuti, G. Iernetti, and M. S. Sagoo, "Optical visualization of non-linear acoustic propagation in cavitating liquids," Ultrasonics 18, 111 (1980).

${ }^{3}$ Yu. A. Kobelev, L. A. Ostrovsky, and A. M. Sutin, "Self-bleaching effect for the acoustic waves in a liquid with gas bubbles," JETP Lett. 30, 395 (1979).

${ }^{4}$ Yu. A. Kobelev and L. A. Ostrovsky, "Nonlinear acoustic phenomena due to bubble drift in a gas-liquid mixture," J. Acoust. Soc. Am. 85, 621 (1989).

${ }^{5}$ S. L. Lopatnikov, "Acoustic phase echo in liquid with gas bubbles," Sov. Tech. Phys. Lett. 6, 270 (1980).

${ }^{6}$ E. Zabolotskaya, "Nonlinear waves in liquid with gas bubbles," Trudi IOFAN 18, 121 (1989).

${ }^{7}$ I. Sh. Akhatov and V. A. Baikov, "Propagation of sound perturbations in heterogeneous gas-liquid systems," J. Eng. Phys. 9, 276 (1986).

${ }^{8}$ I. Sh. Akhatov, V. A. Baikov, and R. A. Baikov, "Propagation of nonlinear waves in gas-liquid media with a gas content variable in space," Fluid Dyn. 7, 161 (1986).

${ }^{9}$ I. Akhatov, U. Parlitz, and W. Lauterborn, "Pattern formation in acoustic cavitation," J. Acoust. Soc. Am. 96, 3627 (1994).

${ }^{10}$ I. Akhatov, U. Parlitz, and W. Lauterborn, "Towards a theory of selforganization phenomena in bubble-liquid mixtures," Phys. Rev. E 54, 4990 (1996).

${ }^{11}$ U. Parlitz, R. Mettin, S. Luther, I. Akhatov, M. Voss, and W. Lauterborn, "Spatiotemporal dynamics of acoustic cavitation bubble clouds," Philos. Trans. R. Soc. London, Ser. A 357, 313 (1999).

${ }^{12}$ O. A. Druzhinin, L. A. Ostrovsky, and A. Prosperetti, "Low-frequency acoustic wave generation in a resonant bubble-layer," J. Acoust. Soc. Am. 100, 3570 (1996).

${ }^{13}$ L. A. Ostrovsky, A. M. Sutin, I. A. Soustova, A. I. Matveyev, and A. I. Potapov, "Nonlinear, low-frequency sound generation in a bubble layer: Theory and laboratory experiment," J. Acoust. Soc. Am. 104, 722 (1998).

${ }^{14} \mathrm{~L}$. van Wijngaarden, "On the equations of motion for mixtures of liquid and gas bubbles," J. Fluid Mech. 33, 465 (1968).

${ }^{15}$ V. E. Nakoryakov, V. G. Pokusaev, and I. R. Shreiber, Propagation of Waves in Gas- and Vapour-liquid Media (Institute of Thermophysics, Novosibirsk, 1983).

${ }^{16}$ S. S. Kutateladze and V. E. Nakoryakov, Heat and Mass-transfer and Waves in Gas-liquid Systems (Nauka, Novosibirsk, 1984).

${ }^{17}$ N. A. Gumerov, "Quasi-monochromatic weakly non-linear waves in a low-dissipative bubbly medium,” J. Appl. Math. Mech. 56, 58 (1992).

${ }^{18}$ D. J. Benney, "Significant interactions between small and large scale surface waves," Stud. Appl. Math. 55, 93 (1976).

${ }^{19}$ D. J. Benney, "A general theory for interactions between short and long waves," Stud. Appl. Math. 56, 81 (1977).

${ }^{20} \mathrm{~V}$. D. Djordjevic and L. G. Redekopp, "On two-dimensional packets of capillary-gravity waves," J. Fluid Mech. 79, 703 (1977).

${ }^{21}$ V. E. Zakharov, “Collapse of Langmuir waves,” Sov. Phys. JETP 72, 908 (1972).

${ }^{22} \mathrm{H}$. Hasimoto and H. Ono, "Nonlinear modulation of gravity waves," J. Phys. Soc. Jpn. 33, 805 (1972).

${ }^{23}$ A. Davey and K. Stewartson, "On three-dimensional packets of surface waves," Proc. R. Soc. London, Ser. A 338, 101 (1974).

${ }^{24}$ J.-M. Chidaglia and J.-C. Saut, "On the initial value problem for the Davey-Stewartson systems," Nonlinearity 3, 475 (1990).

${ }^{25}$ M. J. Ablowitz and H. Segur, "On the evolution of packets of water waves," J. Fluid Mech. 92, 691 (1979).

${ }^{26}$ G. C. Papanicolaou, C. Sulem, P. L. Sulem, and X. R. Wang, "The focusing singularity of the Davey-Stewartson equations for gravity-capillary surface waves," Physica D 72, 61 (1994).

${ }^{27} \mathrm{~S}$. V. Iordansky, "Equations of motion of liquid containing gas bubbles," Zh. Prikl. Mekh. Tekh. Fiz. 102 (May 1960).

${ }^{28}$ V. K. Kedrinsky, "Propagation of small disturbances in liquid containing gas bubbles," Zh. Prikl. Mekh. Tekh. Fiz. 29 (July 1968).

${ }^{29}$ R. I. Nigmatulin, Dynamics of Multiphase Media, Vols. 1 and 2 (Hemisphere, Washington, 1990).

${ }^{30}$ I. Sh. Akhatov and D. B. Khismatullin, "Long-wave-short-wave interaction in bubbly liquids," J. Appl. Math. Mech. 63, 917 (1999).

${ }^{31}$ A. Jeffrey and T. Kawahara, Asymptotic Methods in Nonlinear Wave Theory (Pitman, London, 1982).

${ }^{32}$ R. K. Dodd, J. Eilbeck, J. Gibbon, and H. Morris, Solitons and Nonlinear Wave Equations (Academic, London, 1984).

${ }^{33}$ B. B. Kadomtsev and V. I. Petviashvili, "On the stability of solitary waves in weakly dispersive media," Sov. Phys. Dokl. 15, 539 (1970).

${ }^{34}$ B. G. Konopelchenko, Introduction to Multidimensional Integrable Equations: The Inverse Spectral Transform in $2+1$ Dimensions (Plenum, New York. 1992).

${ }^{35}$ M. J. Ablowitz and P. A. Clarkson, Solitons, Nonlinear Evolution Equations and Inverse Scattering (Cambridge University Press, Cambridge, 1991).

${ }^{36}$ M. Lakshmanan, "Analytic methods for soliton systems," Int. J. Bifurcation Chaos Appl. Sci. Eng. 3, 3 (1993).

${ }^{37}$ D. E. Pelinovsky and Yu. A. Stepanyants, "Self-focusing instability of plane solitons and chains of tow-dimensional solitons in positivedispersion media," Sov. Phys. JETP 77, 602 (1993).

${ }^{38}$ Yu. S. Kivshar and D. E. Pelinovsky, "Self-focusing and transverse instabilities of solitary waves," Phys. Rep. 331, 117 (2000).

${ }^{39}$ E. Infeld, A. Senatorski, and A. A. Skorupski, "Decay of KadomtsevPetviashvili solitons," Phys. Rev. Lett. 72, 1345 (1994).

${ }^{40}$ E. Infeld, A. Senatorski, and A. A. Skorupski, "Numerical simulations of Kadomtsev-Petviashvili soliton interactions," Phys. Rev. E 51, 3183 (1995).

${ }^{41}$ P. Frycz and E. Infeld, "Bending of ion-acoustic solitons," Phys. Rev. A 41, 3375 (1990).

${ }^{42}$ S. Gavrilyuk, "Solitary waves in bubbly liquids are linearly unstable," Eur. J. Mech. B/Fluids 15, 37 (1996).

${ }^{43}$ V. E. Zakharov and A. B. Shabat, "Exact theory of two-dimensional selffocusing and one-dimensional self-modulation of waves in nonlinear media," Sov. Phys. JETP 34, 62 (1972).

${ }^{44}$ T. B. Benjamin and J. E. Feir, "The disintegration of wave trains in deep water," J. Fluid Mech. 27, 417 (1967).

${ }^{45}$ Y.-C. Ma, "The complete solution of the long-wave-short-wave resonance equations," Stud. Appl. Math. 59, 201 (1978).

${ }^{46}$ A. S. Fokas and P. M. Santini, "Dromions and a boundary value problem for the Davey-Stewartson I equation," Physica D 44, 99 (1990).

${ }^{47}$ N. Yoshida, K. Nishinari, J. Satsuma, and K. Abe, "Dromion can be remote-controlled," J. Phys. A 31, 3325 (1998).

${ }^{48}$ S. A. Akhmanov, A. P. Sukhorukov, and R. V. Khokhlov, "Optical selffocusing and diffraction in a nonlinear medium," Usp. Fiz. Nauk 93, 19 (1967).

${ }^{49}$ I. Sh. Akhatov and D. B. Khismatullin, "Mechanisms of interaction between ultrasound and sound in liquids with bubbles: Singular focusing," Acoust. Phys. 47, 140 (2001). 
${ }^{50}$ Yu. A. Berezin, Numerical Modelling of Nonlinear Waves in a Rarefied Plasma (Nauka, Novosibirsk, 1977).

${ }^{51}$ G. I. Marchuk, Methods of Numerical Mathematics (Springer-Verlag, New York, 1975).

${ }^{52}$ C. A. J. Fletcher, Computational Techniques for Fluid Dynamics. 1. Fundamental and General Techniques (Springer-Verlag, Berlin, 1988).

${ }^{53}$ J. L. Bona, V. A. Dougalis, O. A. Karakashian, and W. R. McKinney, "The effect of dissipation on solutions of the generalized Korteweg-de Vries equation," J. Comput. Appl. Math. 74, 127 (1996).

${ }^{54}$ A. M. Sutin, S. W. Yoon, E. J. Kim, and I. N. Didenkulov, "Nonlinear acoustic method for bubble density measurements in water," J. Am. Chem. Soc. 103, 2377 (1998).
${ }^{55}$ M. R. Bailey, D. T. Blackstock, R. O. Cleveland, and L. A. Crum, "Comparison of electrohydraulic lithotripters with rigid and pressure-release ellipsoidal reflectors. II. Cavitation fields," J. Acoust. Soc. Am. 106, 1149 (1999).

${ }^{56}$ P. Huber, P. Peschke, G. Brix, E. W. Hahn, A. Lorenz, U. Tiefenbacher, W. Wannenmacher, and J. Debus, "Synergistic interaction of ultrasonic shock waves and hyperthermia in the Dunning prostate tumor R3327-AT1," Int. J. Cancer 82, 84 (1999).

${ }^{57}$ U. Lauer, E. Bürgelt, Z. Squire, K. Messmer, P. H. Hofschneider, M. Gregor, and M. Delius, "Shock wave permeabilization as a new gene transfer method," Gene Ther. 4, 710 (1997). 